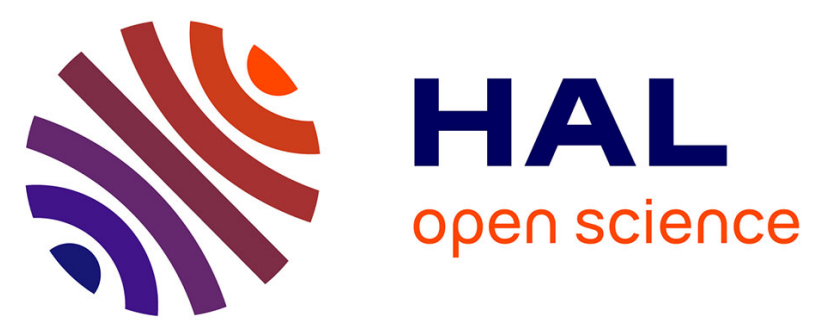

\title{
Continuum modelling of pressure-balanced and fluidized granular flows in 2D: comparison with glass bead experiments and implications for concentrated pyroclastic currents
}

E.C.P. Breard, J. Dufek, Olivier Roche

\section{To cite this version:}

E.C.P. Breard, J. Dufek, Olivier Roche. Continuum modelling of pressure-balanced and fluidized granular flows in 2D: comparison with glass bead experiments and implications for concentrated pyroclastic currents. Journal of Geophysical Research: Solid Earth, 2019, 124, pp.5557-5583. 10.1029/2018JB016874 . hal-02277041

\author{
HAL Id: hal-02277041 \\ https://hal.uca.fr/hal-02277041
}

Submitted on 18 Nov 2021

HAL is a multi-disciplinary open access archive for the deposit and dissemination of scientific research documents, whether they are published or not. The documents may come from teaching and research institutions in France or abroad, or from public or private research centers.
L'archive ouverte pluridisciplinaire HAL, est destinée au dépôt et à la diffusion de documents scientifiques de niveau recherche, publiés ou non, émanant des établissements d'enseignement et de recherche français ou étrangers, des laboratoires publics ou privés.

$$
\text { Copyright }
$$




\author{
RESEARCH ARTICLE \\ 10.1029/2018JB016874 \\ Key Points: \\ - Multiphase modeling of gas-particle \\ flows is validated against \\ experiments \\ - The addition of frictional stresses is \\ required to describe the dense flow \\ rheology \\ - Pore pressure feedback and dynamic \\ pressure control basal pore pressure
}

Correspondence to:

E. C. P. Breard,

ebreard@uoregon.edu

Citation:

Breard, E. C. P., Dufek, J., \& Roche, O. (2019). Continuum modeling of pressure-balanced and fluidized granular flows in 2-D: Comparison with glass bead experiments and implications for concentrated pyroclastic density currents. Journal of Geophysical Research: Solid Earth, 124 5557-5583. https://doi.org/10.1029/ 2018JB016874

Received 15 OCT 2018

Accepted 21 MAY 2019

Accepted article online 29 MAY 2019

Published online 23 JUN 2019
(C)2019. American Geophysical Union. All Rights Reserved.
Continuum Modeling of Pressure-Balanced and Fluidized Granular Flows in 2-D: Comparison With Glass Bead Experiments and Implications for Concentrated Pyroclastic Density Currents

\author{
E. C. P. Breard ${ }^{1}$ iD, J. Dufek ${ }^{1}$ (D), and O. Roche ${ }^{2}$ iD \\ ${ }^{1}$ Department of Earth Sciences, University of Oregon, Eugene, OR, USA, ${ }^{2}$ Laboratoire Magmas et Volcans, Université \\ Clermont Auvergne-CNRS-IRD, OPGC, Aubière, France
}

\section{Introduction}

\subsection{Problem Addressed}

Pyroclastic density currents (PDCs) display a wide range of particle concentrations (Breard et al., 2016; Druitt, 1998; Dufek, 2016; Sulpizio et al., 2014), which imply a commensurately large range of gas-particle and particle-particle interactions. The full complement of interactions is also often referred to as four-way coupling (Balachandar \& Eaton, 2010). The occurrence of four-way coupling implies that granular stresses cannot be neglected. These stresses can be modeled in gas-particle systems, either by discrete element modeling (DEM) or by continuum modeling where both gas and particulate phases are represented as distinct continua (multiphase) or as a mixed continuum (single-phase approximation). However, DEM is only feasible for systems with relatively limited number of particles (typically <20 million in 2018) owing to present computational limitations. Therefore, we here focus on the multiphase continuum approach that can be used for modeling natural systems as well. Complicating the analysis of PDC is the ability of these currents to develop elevated pore fluid (gas) pressure and to be either fully or partially fluidized, thus enhancing transport. Here we refer to "pressure-balanced" granular systems in the case where the internal gas pressure is close to atmospheric pressure, whereas a system involving momentum exchange as a result of elevated pore fluid pressure is referred to as "fluidized."

Pressure-balanced granular flows (including monodisperse mixtures) have been treated through modeled granular rheologies that range from relatively simple (Fullard et al., 2017) to complex (Forterre \& Pouliquen, 2008; Goddard, 2014). While the exchange of forces is limited to direct transmission between 
grains, the interactions between numerous grains and their collective rheology is unclear and is a matter of active study (Amarsid et al., 2017; Boyer et al., 2011). This complexity is a consequence of local (da Cruz et al., 2005) and nonlocal interactions (Kamrin \& Koval, 2012), as well as history and preparation-dependent dilation and strengthening (Wood, 1990; Roux \& Radjai, 1998; Kamrin, 2017), which in turn depends on particle size distribution, particle stiffness, friction coefficients, and particle shape (Azéma et al., 2012; Du et al., 2016; GDR-MiDi, 2004; Rognon et al., 2006).

The present work aims at testing a multiphase formulation on conditions relevant for concentrated pyroclastic density currents (Charbonnier \& Gertisser, 2011; Roche, 2012; Breard \& Lube, 2017). Continuum multiphase models have been used widely both in industry and applied to natural flows including turbidity currents (Meiburg \& Kneller, 2010) and snow avalanches (Bartelt et al., 2017). Here we use the MFIX code base to examine different rheological models in a continuum multiphase approach (Syamlal \& O'Brien, 1993). MFIX has been used by several authors and applied across a broad range of particle concentrations. Modified variants of MFIX have been used to model mixtures with a range of particle concentrations that develop in pyroclastic density currents as a result of entrainment, sedimentation, and turbulent sorting (Dufek, 2016). However, little work has been done on the concentrated end of the spectrum, which dominates the dynamics of concentrated regions of PDCs (e.g., basal parts of the density current; Maurin et al., 2015) and are often neglected when dealing with large dilute turbulent currents (Esposti Ongaro et al., 2012). Simulations are compared with well-constrained benchtop experiments (Roche et al., 2010). First, we give a brief overview of the current state-of-the art of the granular physics with respect to continuum models of granular flow and present how granular stresses are modeled in a continuum multiphase framework. Then, we present simulation results of monodisperse pressure-balanced and fluidized dam-break collapses and focus on the pore pressure evolution. Finally, we show simulations of bidisperse pressurebalanced and fluidized cases and discuss implications for volcanic flows.

\subsection{Brief Review of Continuum Granular Models}

Granular media can be classified into four different regimes (Jaeger et al., 1996): (1) a dense quasi-static regime wherein particles interact by frictional contacts (Roux \& Combe, 2002), (2) an intermediate liquidlike regime wherein particles interact both by binary collision and friction (GDR-MiDi, 2004; Pouliquen \& Chevoir, 2002), (3) a "dilute" gaseous regime wherein the particles interact by binary collision, and (4) a drag dominated regime, where particle-particle interactions are minimal (Goldhirsch, 2003). These interactions are inclusive of the particle and fluid interactions examined in this work, and in particular we note that the physics of granular flows described in this section applies also to fluidized beds (that is, flows with pore fluid pressure gradients). In particular, all solid stresses are described similarly ( Syamlal et al., 2016) because particle interactions are described in the same way but are mitigated by particle-fluid interactions.

No current theory can describe granular flows across all regimes and solid fractions, even in steady homogenous settings (such as time and spatial invariant properties such as shear rate and solid concentration). Approaches suited toward one regime often fail to accurately describe dynamics at other particle concentrations. For example, the "gaseous" regime of granular flows is often described by kinetic theory (KT) that has been derived by extending the KT of nonuniform gases which assumes inelastic binary collisions of particles (J. T. Jenkins \& Savage, 1983; Lun et al., 1984). In these systems kinetic energy dissipates during the binary collisions, which, as opposed to elastic gaseous molecules, are inelastic. A transport equation for granular temperature (variance in the particle velocity distribution) is usually developed to reconstruct the probability of collisions (and momentum transfer). This assumption (binary collisions) is satisfied in "relatively" dilute regimes with particle volumetric concentration (EP_S) $<0.49-0.5$ (Chialvo \& Sundaresan, 2013). This theory has been tested against DEM simulations and gives good agreement in these regimes (Chialvo \& Sundaresan, 2013; Duan et al., 2017). One of the successes of KT is the reconstruction of macroscale transport relations from a probabilistic model of granular encounters in the microscale. However, in the denser regime (volume fraction $>0.5$ ), where multiparticle enduring frictional contacts also exist, KT simulations and DEM simulations results diverge. Therefore, in recent years, much effort has been devoted to extend the applicable range of KT to denser regimes (Chialvo \& Sundaresan, 2013; Duan et al., 2017; Vescovi et al., 2014). Some authors proposed adding a length scale parameter to enhance the energy dissipation rate based on longer range interactions in more concentrated flows (Jenkins \& Berzi, 2010), while others modified the radial distribution function at contact (RDF) with the addition of a force chain length scale and effective restitution 
coefficient (Chialvo \& Sundaresan, 2013). For the latter, Chialvo and Sundaresan (2013) stipulate that the modified KT is only applicable for steady and uniform granular flows (e.g., hopper mass flow discharge). Duan et al. (2017) proposed the extended KT that incorporates energy dissipation due to contact length, which in turn depends on particle stiffness. Their simulations suggest that the extended KT extends the viability of the KT up to particle volumetric concentration of 0.57 (for monodisperse distributions).

For concentration larger than 0.57 , in the intermediate and quasi-static regimes, granular flows have been modeled following different approaches (frictional plasticity, inertial rheology, and nonlocal granular fluidity [NGF]; Jop et al., 2006; Zhang \& Kamrin, 2017) that replace the description of stresses accounted by the KT. Three models are described below from simplest to most complex:

\subsubsection{Frictional Plasticity}

This model comprises incompressibility and the Drucker-Prager failure criterion (Drucker \& Prager, 1952).

Where transient effects are negligible, the Drucker-Prager failure criterion can be expressed as

$$
\mu_{\text {static }}=\frac{\tau}{p} \text { if } \dot{\gamma}>0 \text { and } \tau \leq \mu_{\text {static }} p
$$

where $\tau$ is the shear stress, $\dot{\gamma}$ is the shear rate, $p$ is the solid pressure (normal stress), and $\mu_{\text {static }}$ is the internal static friction coefficient, which is assumed to be constant. To ensure the nonnegativity of the pressure (no tension), the following criterion is used:

$$
p=0 \text { if } C<C_{c}
$$

where $C_{c}$ is the critical packing fraction that imposes the onset of contact between grains. Beyond this packing fraction, the flow is modeled as stress free (e.g., granular gas). This differs from plastic dilation in that particles support pressure while dilating.

\subsubsection{Inertial Rheology}

Following the work of GDR-MiDi (2004) and da Cruz et al. (2005), the inertial number I, also known as the square root of the Savage number (Savage, 1998), controls the granular rheology and is defined as

$$
I=\dot{\gamma} \sqrt{\frac{\rho_{s} d^{2}}{p}}
$$

with $\rho_{s}$ and $d$ as the particle density and diameter, respectively. The effect of shear upon the plastic flow is essential. This was first observed by Bagnold in rapidly sheared granular flows (Bagnold, 1954) but was further investigated only in the past decades (Schaeffer, 1987). It has been shown for steady homogeneous flows that the friction coefficient is a function of the inertial number as

$$
\mu(I)=\mu_{\text {static }}+\frac{\mu_{d}-\mu_{\text {static }}}{\left(\frac{I_{0}}{I}+1\right)}
$$

$\mu_{d}$ is the friction coefficient that $\mu(I)$ tends to as $I$ increases, and $I_{o}$ is a dimensionless parameter that depends on material property. To ensure well-posedness of the constitutive equations derived from equation (4), the upper formula is only valid across a small range of Inertial number (e.g., $0.001-0.3$ for monodisperse glass beads; Barker et al., 2017). Well-posedness ensures that the solution is stable, as opposed to ill-posedness that could lead to instabilities that depend for instance on the grid size spacing. This led Barker et al. (2017) to propose a modified friction coefficient:

$$
\begin{aligned}
& \mu(I)=\left(\begin{array}{ll}
\sqrt{\frac{\alpha}{\ln \left(\frac{A_{1}}{I}\right)}} & I \leq I_{1}^{N} \\
\frac{\mu_{\text {static }} I_{0}+\mu_{d} I+\mu_{\infty} I^{2}}{I_{0}+I} & I>I_{1}^{N}
\end{array}\right) \\
& A_{1}=I_{1}^{N} \exp \left(\frac{\alpha\left(I_{0}+I_{1}^{N}\right)^{2}}{\left(\mu_{\text {static }} I_{0}+\mu_{d} I_{1}^{N}+\mu_{\infty}\left(I_{1}^{N}\right)^{2}\right)^{2}}\right)
\end{aligned}
$$

where $I_{0}$ (same as in equation (4)), $\mu_{\infty}$, and $\alpha$ are constants. $I_{1}^{N}$ is the lower end of $I$ where equation (4) is well- 
posed. Equation (5) ensures well-posedness for $I=0$ to $\sim 10$. The form of the friction coefficient at high inertial number is based on the experimental work of Holyoake and McElwaine (2012). Note that the $\mu(I)$ theory implies that at slopes greater than the angle of repose $\mu_{\text {static }}$, the granular flow can reach steady state (Brodu et al., 2015). Without the dependence on $I$, the flow would accelerate continuously.

\subsubsection{NGF}

In many settings, granular flows are neither steady and/or homogenous, and the inertial rheology, which depends locally on $I$ does not reproduce observed dynamics. This is exemplified in cases with heterogeneous strain rate fields, where the yield criterion imposed by the material properties does not apply everywhere, as portions of the flow with $\mu<\mu_{\text {static }}$ remain flowing (Dunatunga \& Kamrin, 2015). Additionally, Pouliquen (1999) noted that thinner flows required higher slopes to come to rest, whereas the friction coefficient was thought to be equal the tangent of the slope angle irrespective of the flow thickness. This discrepancy may be due to friction at the lateral walls of the experimental channel (Brodu et al., 2015). Kamrin and Koval (2012) has shown that cooperativity of particle motion is missing in other rheology descriptions (i.e., Jop et al., 2006). In short, the local flow mechanics is dependent on the flow properties taking place at other locations (termed nonlocal or cooperative interactions). Thus, a model called "granular fluidity" was introduced to capture the importance of another length-scale describing the cooperativity. This NGF model is rooted in the introduction of a scalar state field named granular fluidity and denoted as $g(x)$, which exists throughout the granular media. This " $g$ " field is a kinematically observable state variable (Zhang \& Kamrin, 2017) that is defined by a reaction-diffusion form equation, similar to that of Landau-type equation:

$$
t_{0} \dot{g}=A^{2} d^{2} \nabla^{2} g-\mu\left(\frac{\mu_{s}-\mu}{\mu_{2}-\mu}\right) g-b \sqrt{\frac{\rho_{s} d^{2}}{p}} \mu g^{2}
$$

wherein $t_{0}$ is a constant time scale and $\mu_{2}=\mu_{\text {static }}+\mu$. $\mu$ and $b$ are material constants. The $g$ field correlates the strain rate and stress as

$$
\mu=\frac{\dot{\gamma}}{g}
$$

$\mu$ is the ratio of shear stress and normal stress. The granular fluidity $g$ has the dimensions of per second. $g=0$ if $\mu<\mu_{\text {static }}$, while $g$ is $>0$ when $\mu>\mu_{\text {static. }}$ Regions where $g$ is large act as a fluidity source and diffuse into regions of lower fluidity, allowing the material to flow. This theory explains rheological measurements of friction coefficient lower than $\mu_{s}$ at low inertial number for systems where shear rates are heterogeneous across the sample (Kamrin \& Koval, 2012). It also predicts that, as the flow thins $(h / d$ decreases), the friction coefficient at a given shear rate increases. Using the NGS model, one can predict the flow fields even in steady conditions as it returns to the inertial theory. Most importantly, the NGS model predicts the flow field of creeping flows, where local inertial rheology fails.

Typically, granular flow models neglect the interstitial fluid and its interaction with particles. However, in concentrated pyroclastic density currents with high amounts of fine particles (down to microns size), gas most likely plays an important role in controlling the flow dynamics, meaning that drag forces are nonnegligible. Additionally, elevated pore pressure may arise because of gas entrapment during their generation mechanism (i.e., column collapse, (Breard et al., 2017; Rowley et al., 2014; Sweeney \& Valentine, 2017; Valentine \& Sweeney, 2018), gas exsolution during transport (Sparks, 1976, 1978), and ongoing incorporation of air (e.g., Benage et al., 2016). No internal measurements of pore pressure have hitherto been achieved; thus, the presence of elevated pore pressure in PDCs remains the hypothesis that we favor.

\subsection{MFIX Continuum Model}

In the following section, we detail the model used in this paper where gas-particles flows are modeled as two continua phases that exchange momentum. We examine the implications of collisional and frictional solid stresses using the open source MFIX code. The MFiX (Multiphase Flow with Interphase eXchanges) suite is a set of multiphase computational fluid dynamics models for describing the hydrodynamics, heat transfer, and chemical reaction in dilute and dense multiphase mixtures (Syamlal et al., 2016). MFiX development has been ongoing at National Energy Technology Laboratory for over two decades, beginning with a multiparticle algorithm (Syamlal, 1985) based on the Eulerian-Eulerian approach. During past years, MFiX has undergone continuous model development, verification and validation, and a wide range of application 
(Benyahia et al., 2007; Benyahia, 2008; Bergantz et al., 2015; Dartevelle, 2007; Dartevelle \& Valentine, 2008; Dufek \& Bergantz, 2005; Li et al., 2017; Srivastava \& Sundaresan, 2003; M Syamlal et al., 2017). In this study we focus on the MFIX-TFM (two-fluid) model package, which describes the motion of a mixture of gas (or liquid) and solids as two or more continuum phases. Due to the continuum representation of the particles, the individual motion of particles or gas around particles is not modeled, which in turn reduces the computational cost by orders of magnitudes (Syamlal \& O'Brien, 1993; Syamlal \& Pannala, 2011). However, such continuum models require constitutive equations describing gas-solid drag and solid stress. The total stresses that apply to the solid phase are the sum of collisional $\tau_{k}$ and frictional stresses $\tau_{f}$. The former stresses are derived from the KT, while the later are derived using the Schaeffer frictional model (Schaeffer, 1987) or Princeton frictional model of Srivastava-Sundaresan (Srivastava \& Sundaresan, 2003). The conservation of mass for constant solid density is defined as

$$
\rho_{s}\left(\frac{\partial \varepsilon_{s}}{\partial t}+\nabla \cdot\left(\varepsilon_{s} v_{s}\right)\right)=0
$$

where $\varepsilon_{s}$ is the solid volume fraction, $\rho_{s}$ is the solid density, and $v_{s}$ is the solid velocity vector. The conservation of linear momentum is given by

$$
\rho_{s}\left(\frac{\partial \varepsilon_{s} v_{s}}{\partial t}+\nabla \cdot\left(\varepsilon_{s} v_{s} v_{s}\right)\right)=\nabla \cdot\left(\tau_{k}+\tau_{f}\right)+\varepsilon_{s} \rho_{s} g
$$

with $\tau_{k}$ and $\tau_{f}$ the kinetic and friction stress tensors, respectively, and $g$ is the gravitational acceleration. The translational granular energy conservation equation is

$$
\frac{3}{2} \rho_{s}\left(\frac{\partial \varepsilon_{s} \theta_{s}}{\partial t}+\nabla \cdot\left(\varepsilon_{s} \theta_{s} v_{s}\right)\right)=-\nabla \cdot q+\tau_{k}: \nabla v_{s}-\rho_{s} J_{s}
$$

where $\theta_{s}$ is the granular temperature, and $J_{S}$ is the granular energy dissipation due to inelastic collisions. Solid kinetic-collisional and frictional stress terms are defined as

$$
\begin{gathered}
\tau_{k}=\left(-P_{s}+\eta \mu_{b} \nabla \cdot v_{s}\right) I+2 \mu_{s} S_{s} \\
\tau_{f}=-P_{f} I+2 \mu_{f} S_{s} \\
S_{s}=\frac{1}{2}\left(\nabla v_{s}+\left(\nabla v_{s}\right)^{T}\right)-\frac{1}{3} \nabla \cdot v_{s} I
\end{gathered}
$$

where $P_{S}$ is the solid pressure in viscous regime (that corresponds to the pressure due to kinetic interactions $=\mathrm{KT}), P_{f}$ is the solid pressure in frictional regime, $S_{s}$ is the strain rate tensor, $\eta$ equals $(1+e) / 2$ with $e$ as restitution coefficient, $\mu_{b}$ is the bulk viscosity of the solid phase, and $I$ is the identity tensor. The solid pressure in viscous regime is defined as

$$
P_{s}=\varepsilon_{s} \rho_{s} \theta_{s}\left[1+4 \eta \varepsilon_{s} g_{o}\right]
$$

The RDF at contact " $g_{o}$ " is

$$
g_{o}=\frac{1-0.5 \varepsilon_{s}}{\left(1-\varepsilon_{s}\right)^{3}}
$$

(Carnahan \& Starling, 1969).

The solid viscosity $\mu_{s}$ is expressed as

$$
\mu_{s}=\left(\frac{2+\alpha}{3}\right)\left[\frac{\mu}{g_{o} \eta(2-\eta)}\left(1+\frac{8}{5} \eta \varepsilon_{s} g_{o}\right)\left(1+\frac{8}{5} \eta(3 \eta-2) \varepsilon_{s} g_{o}+\frac{8}{5} \eta \mu_{b}\right)\right]
$$

with $\alpha$ a constant $=1.6$ based on the KT (Lun et al., 1984), $\mu_{b}$ the bulk viscosity of the solid, $\eta=(1+e) / 2$ with $e$ as the particle-particle restitution coefficient, and $\mu$ is the solid phase dilute granular viscosity defined as 


$$
\mu=\frac{5}{96} \rho_{s} d_{p} \sqrt{\pi \theta_{s}}, \mu_{b}=\frac{256}{5 \pi} \mu \varepsilon_{s} g_{o}^{2}
$$

The granular energy flux and conductivity are given by

$$
\begin{gathered}
q=-\kappa_{s} \nabla \theta_{s} \\
\text { with } \kappa_{s}=\frac{\kappa}{g_{o}}\left[\left(1+\frac{12}{5} \eta \varepsilon_{s} g_{o}\right)\left(1+\frac{12}{5} \eta^{2}(4 \eta-3) \varepsilon_{s} g_{o}\right)+\frac{64}{25 \pi}(41-33 \eta) \eta^{2}\left(\varepsilon_{s} g_{o}\right)^{2}\right] \\
\kappa=\frac{75 \rho_{s} d_{p} \sqrt{\pi \theta_{s}}}{48 \eta(41-33 \eta)}
\end{gathered}
$$

The collisional dissipation of granular energy is

$$
J_{s}=\frac{48}{\sqrt{\pi}} \eta(1-\eta) \frac{\varepsilon_{s}^{2} g_{o}}{d_{p}} \theta_{s}^{3 / 2}
$$

and the frictional-collisional wall boundary condition is

$$
\begin{gathered}
\frac{v_{s l}}{\left|v_{s l}\right|} \cdot\left(\tau_{k}+\tau_{f}\right) \cdot n+\frac{\varnothing \pi \rho_{s} \varepsilon_{s} g_{o} \sqrt{\theta_{s}}}{2 \sqrt{3} \varepsilon_{s} \max } v_{s l}+\left(n \cdot \tau_{f} \cdot n\right) \tan \delta=0 \\
n . q=\frac{\varnothing \pi\left|v_{s}\right|^{2} \rho_{s} \varepsilon_{s} g_{o} \sqrt{\theta_{s}}}{2 \sqrt{3} \varepsilon_{s} \max }-\frac{\sqrt{3} \pi \rho_{s} \varepsilon_{s} g_{o}\left(1-e_{w}{ }^{2}\right) \sqrt{\theta_{s}}}{4 \varepsilon_{s}{ }^{\max }} \theta_{s}
\end{gathered}
$$

where $\varnothing$ is the specularity coefficient $\Theta^{*}$, which is an empirical parameter qualifying the nature of particlewall collisions and ranges from 0 for perfect specular collisions to 1 for perfectly diffuse collisions; $n$ is the unit vector normal to the wall surface; $q$ is the flux of granular energy, $\varepsilon_{s}$ max is the maximum solid fraction, $e_{w}$ is the wall restitution coefficient, and $\delta$ is the angle of internal friction.

There are two implemented frictional models: (1) the Schaeffer and (2) Princeton models, used in simulations presented in this paper.

The Schaeffer model describes the plastic flow of a granular media, which occurs once the volume fraction exceeds the maximum packing. This model allows for slight compressibility near the packing limit $\varepsilon_{S}^{\max }$ and is inspired from plastic theories (Jenike, 1987; Tardos, 1997). The frictional stresses and pressure are expressed as

$$
\begin{aligned}
& P_{f}=P_{c}= \begin{cases}10^{25}\left(\varepsilon_{S}-\varepsilon_{S}{ }^{\max }\right)^{10} & \text { when } \varepsilon_{S}>\varepsilon_{S}{ }^{\max } \\
0 & \text { when } \varepsilon_{S} \leq \varepsilon_{S}{ }^{\max }\end{cases} \\
& \mu_{f} \begin{cases}P_{c} \sin \delta & \text { when } \varepsilon_{s}>\varepsilon_{s}^{\max } \\
0 & \text { when } \quad \varepsilon_{s} \leq \varepsilon_{s}^{\max }\end{cases}
\end{aligned}
$$

where $P_{c}$ is the critical state pressure.

This model uses the second invariant of the deviator of the strain rate tensor $\|D\|$ that is defined as follows in 3-D:

$$
\|D\|=\frac{1}{6}\left[\left(D_{s, 11}-D_{s, 22}\right)^{2}+\left(D_{s, 22}-D_{s, 33}\right)^{2}+\left(D_{s, 33}-D_{s, 11}\right)^{2}\right]+D_{s, 12}^{2}+D_{s, 23}^{2}+D_{s, 31}^{2}
$$

where

$$
D_{s, i j}=\frac{1}{2}\left(\frac{\partial u_{s, i}}{\partial x_{i}}+\frac{\partial_{s, j}}{\partial x_{i}}\right)
$$

The Princeton model describes how frictional stresses influence the granular assembly above a minimum solid packing fraction (e.g., 0.58) that is below the maximum random packing fraction $(=0.63-0.64$ for 
monodisperse spheres). This threshold was proposed by Johnson and Jackson (1987). The critical state theory solely applies when the granular media is incompressible (i.e., above maximum packing). Additionally, the Princeton model avoids singularity, even when the quasi-static flow is steady, by incorporating granular temperature and its effect on strain rate fluctuations (Savage, 1998). The model can be expressed as

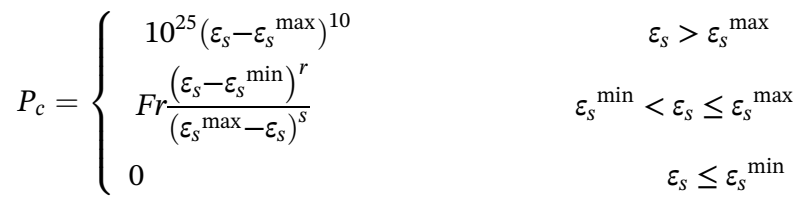

$$
\begin{aligned}
& \frac{P_{f}}{P_{c}}=\left[1-\frac{\nabla \cdot v_{s}}{N \sqrt{2} \sin (\delta) \sqrt{S_{s}: S_{s}+\frac{\theta_{s}}{d^{2}}}}\right]^{N-1} \\
& \mu_{f}=\frac{\sin \delta}{\sqrt{2}} \frac{P_{f}}{\sqrt{S_{s}: S_{s}+\frac{\theta_{s}}{d^{2}}}}\left\{N-(N-1)\left[\frac{P_{f}}{P_{c}}\right]^{\frac{1}{N-1}}\right\}
\end{aligned}
$$

The coefficient $N$ is the exponent that defines the shape of the yield surface and takes the values of $N=\frac{\sqrt{3}}{2}$ $\sin \delta$ for $\nabla . v_{s} \geq 0$ (dilation) or $N=1.3$ when $\nabla . v_{s}<0$ (compaction). Fr is a constant $=0.5 \mathrm{dyn} / \mathrm{cm}^{2}(=0.05$ $\mathrm{Pa}) . S_{s}$ is the strain rate tensor, where the notation $S_{s}: S_{s}=S_{s i j} S_{s i j}$. This model was used in 2-D to simulate the discharge of a bin (Srivastava \& Sundaresan, 2003) and validated against Berverloo correlation for hopper discharge (Beverloo et al., 1961) and Couette shear cell settings (Benyahia, 2008). Based upon the recent work on KT theory, we modified the RDF at contact by the following form:

$$
\begin{array}{r}
g_{o}=f \frac{1-0.5 \varepsilon_{s}}{\left(1-\varepsilon_{s}\right)^{3}}+(1-f) \frac{2}{\varepsilon_{s}{ }^{\max }-\varepsilon_{s}} \\
f= \begin{cases}1 & \varepsilon_{s}<\varepsilon_{m} \\
\frac{\varepsilon_{s}-2 \varepsilon_{m}+\varepsilon_{s}{ }^{\max }\left(2 \varepsilon_{s}-\varepsilon_{s}{ }^{\max }\right)}{2 \varepsilon_{s}{ }^{\max }-\varepsilon_{m}{ }^{2}-\varepsilon_{s}{ }^{\max 2}} & \varepsilon_{s} \geq \varepsilon_{m}\end{cases}
\end{array}
$$

where $\varepsilon_{m}=0.4, \varepsilon_{s}^{\max }=0.64$ (Vescovi et al., 2014). The RDF needs to be modified when dealing with irregularly shaped particles to account for the variation in dissipation of energy with respect to spherical particles (Cagnoli \& Piersanti, 2015).

\section{Methods}

In this paper we investigate transient granular flows, which are unsteady and experience acceleration and deceleration, in both initially pressure-balanced versus fully fluidized conditions. Motivated by small-scale experiments (Roche et al., 2008, 2010), we model a 2-D dam-break collapse of a granular material. In experiments, columns of either monodisperse (one grain size) or bidisperse (two grain sizes) mixtures of glass beads of density $2,500 \mathrm{~kg} / \mathrm{m}^{3}$ were released from a reservoir of width $0.2 \mathrm{~m}$ into a horizontal channel of length $3 \mathrm{~m}$. The granular columns were initially either pressure-balanced or fluidized by an air flow passing through a basal porous plate. Their heights were up to $0.4 \mathrm{~m}$, and their solid volume fraction was $\sim 0.58$ when they were not expanded through fluidization. The columns were released rapidly by means of a sliding gate in order to generate gravitational flows that propagated in the channel. The flows were filmed with a highspeed video camera, and their basal pore pressure was measured by sensors inserted into the channel base at various distances from the reservoir.

\subsection{Monodisperse Flows}

We conducted seven different simulations (four pressure balanced and three fluidized simulations) involving monodisperse mixtures. In experiments, the particles had a very well sorted size distribution (Figure $1 \mathrm{a}^{\prime}$ ), where the Folk and Ward (1957) mean and sorting were 75.5 and $1.2 \mu \mathrm{m}$, respectively, and the Sauter mean diameter was $75 \mu \mathrm{m}$ (Sauter mean $=1 / \sum \frac{x_{i}}{d_{i}}$, with $x_{i}$ as the mass fraction of the grain size 
a

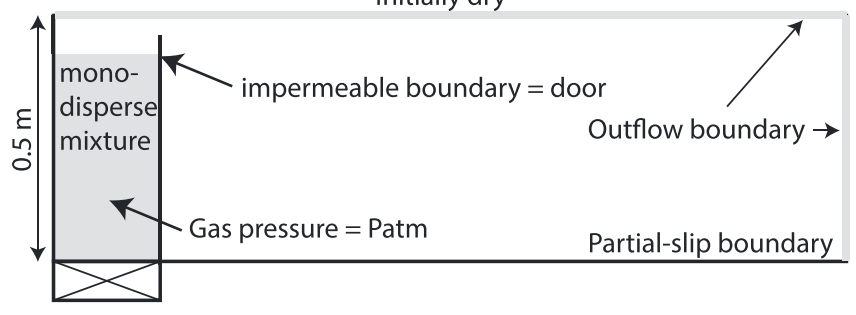

Initially Fluidized
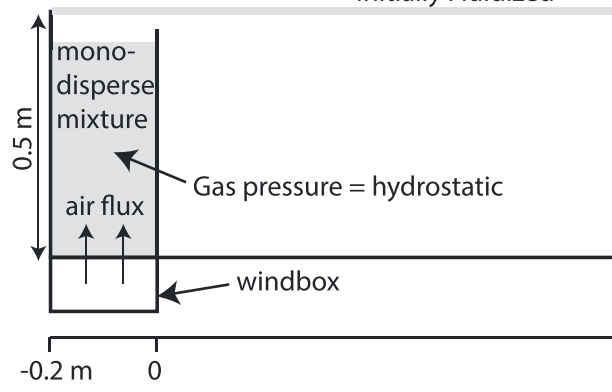

b
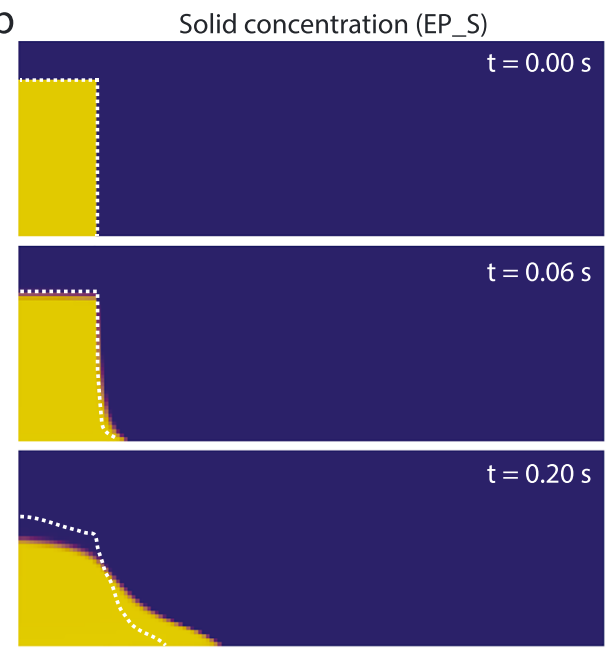

$\mathrm{t}=0.36 \mathrm{~s}$

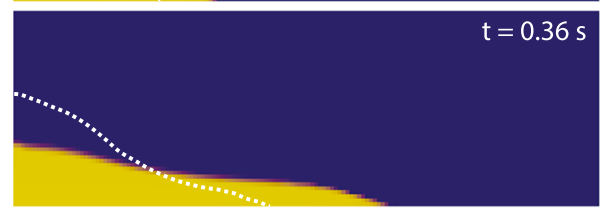

$\mathrm{t}=0.52 \mathrm{~s}$

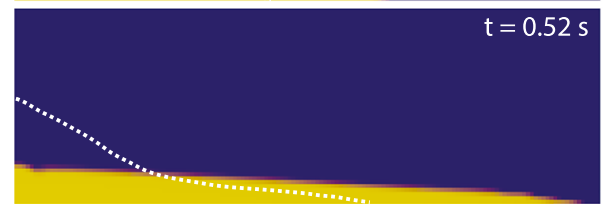

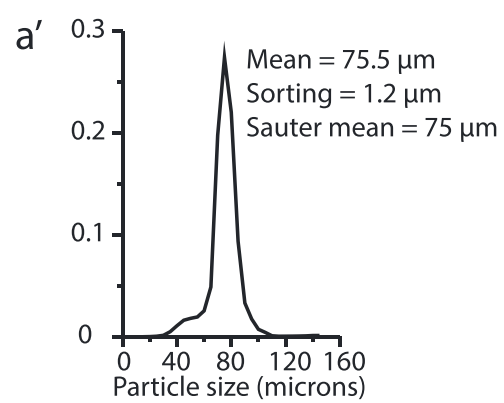

Outflow boundary

Partial-slip boundary

Distance to reservoir gate

$1.2 \mathrm{~m}$

C

Gas pressure (EP_G)

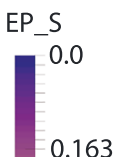

0.163

$-0.325$

0.487

0.65

P_G $(\mathrm{kPa})$

4.0

3.0

$t=0.36 s$

2.0

1.0

$\mathrm{t}=0.52 \mathrm{~s}$

0.0

Figure 1. (a) Sketch of the experimental setup for initially pressure-balanced and fluidized cases and simulation domain. $\left(a^{\prime}\right)$ Grain-size distribution of the experimental mixture determined with a Malvern G3 analyzer. (b) Plot of the solid fraction (EP_S) at various time steps for the simulation using only the kinetic theory to describe solid stresses. The dotted line represents the flow free surface from experiments of Roche et al. (2010). (c) Plot of the gas pore pressure at same time steps as in (b). 
Table 1

Summary of Simulation Parameters

\begin{tabular}{|c|c|c|c|c|c|}
\hline Parameter (units) & Variables & Monodisperse dry & Monodisperse fluidized & Bidisperse (0.4) & Bidisperse (0-1) \\
\hline Domain resolution (m) & $\mathrm{d} x=\mathrm{d} y$ & 0.01 and 0.005 & 0.005 & 0.005 & 0.01 \\
\hline Fluid density $\left(\mathrm{kg} / \mathrm{m}^{3}\right)$ & $\rho_{f}$ & 1.2 & 1.2 & 1.2 & 1.2 \\
\hline Fluid dynamic viscosity ( $\mathrm{Pa} \mathrm{s}$ ) & $\mu_{f}$ & $1.8 \times 10^{-5}$ & $1.8 \times 10^{-5}$ & $1.8 \times 10^{-5}$ & $1.8 \times 10^{-5}$ \\
\hline Particle density $\left(\mathrm{kg} / \mathrm{m}^{3}\right)$ & $\rho_{P}$ & 2500 & & & \\
\hline Particle diameter $(\mathrm{m})$ & $\mathrm{d}$ & $75 \times 10^{-6}$ & $75 \times 10^{-6}$ & $75 \times 10^{-6} 700 \times 10^{-6}$ & $75 \times 10^{-6} 700 \times 10^{-6}$ \\
\hline Angle of internal friction (deg) & $\delta$ & 28 & 28 & 2821 & 2821 \\
\hline Angle of wall friction (deg) & $\delta_{w}$ & 12 & 12 & 12 & 12 \\
\hline Specularity coefficient & $\Theta^{*}$ & 0.25 & 0.25 & 0.25 & 0.25 \\
\hline Particle-wall restitution coefficient & $e_{w}$ & 0.8 & 0.8 & 0.8 & 0.8 \\
\hline Particle-particle restitution coefficient & $e_{n}$ & 0.9 & 0.9 & 0.9 & 0.9 \\
\hline Particle-wall coefficient of friction & $\mu_{w}$ & 0.7 & 0.7 & 0.7 & 0.7 \\
\hline Max packing fraction & $\varepsilon_{s}^{\max }$ & 0.64 & 0.64 & 0.64 & 0.64 \\
\hline Minimum packing for frictional model & $\varepsilon_{S}^{\min }$ & 0.49 & 0.49 & 0.49 & 0.49 \\
\hline Initial packing fraction & EP_S_start & 0.6 & 0.58 & 0.57 & 0.6 \\
\hline Granular temperature $\left(\mathrm{m}^{2} / \mathrm{s}^{2}\right)$ & $\Theta \mathrm{s}$ & 0.01 & 0.01 & 0.01 & 0.01 \\
\hline
\end{tabular}

Note. The slip coefficient is expressed by the specularity coefficient $\Theta^{*}$, which is an empirical parameter qualifying the nature of particle-wall collisions and ranges from 0 for perfect specular collisions to 1 for perfectly diffuse collisions, and depends on the wall roughness.

diameter $d_{i}$ ). Therefore, we chose to represent the distribution as a single grain size of $75 \mu \mathrm{m}$ (Sauter mean), which described best the flow permeability. The parameters of the monodisperse simulations are detailed in Table 1. The static friction coefficient reported was $0.53\left(\tan 28^{\circ}\right.$; Roche et al., 2010), which was higher than others have reported for quasi-monodisperse 1-mm glass beads (friction coefficient $=0.4$; Jop et al., 2006). DEM simulations showed that the static friction coefficient, approximated as the tangent of the angle of repose, was inversely correlated to grain size (Zhou et al., 2001) for all other properties being equal (i.e., particle-particle friction coefficient, stiffness, and restitution coefficients). The simulation domain was 2.5 $\mathrm{m}$ long with a $0.2-\mathrm{m}$ wide reservoir and $0.5 \mathrm{~m}$ in height (with a bed height of $0.4 \mathrm{~m}$ ). The reservoir had a porous plate at its base allowing gas to stream through the bed to achieve static fluidization, as in experiments (Figure 1a).

The fine mesh $(5 \mathrm{~mm})$ was used to effectively resolve variations in the velocities at the boundaries and at the flow front. The initial granular temperature was taken as $0.01 \mathrm{~m}^{2} / \mathrm{s}^{2}$ across the solid (Table 1), as the Princeton model requires $S_{s}: S_{s}+\frac{\theta_{s}}{d^{2}}$ to be $>0$ at all locations. Note that the results were not sensitive to the choice of initial granular temperature.

The boundary condition for the solid phase was taken as the Johson and Jackson (1987) model, which is a frictional-collisional boundary allowing for partial slip. The upper and right boundaries were chosen as open (where pressure was maintained at a constant level throughout the simulation). In simulations, we used an impermeable boundary to represent the door located at $0 \mathrm{~m}(=0.2 \mathrm{~m}$ when 0 is the left boundary of domain). The time $=0 \mathrm{~s}$, represented the time where the reservoir door opened, which was simulated as instantaneous.

For fluidized simulations, we fully fluidized the mixture at the onset of bubbling (Umb in Roche et al., 2010) and left the air influx on at the lower boundary (Figure 1). Due to slight expansion of the bed (due to fluidization) of 3.3\% (compared to the pressure-balanced case), the bed height was $0.413 \mathrm{~m}$. Simulations were repeated with identical conditions, and the results were systematically identical (volume fraction, flow front, and velocities had less than $1 \%$ variability).

\subsection{Bidisperse}

For the set of three bidisperse simulations we blended particles of $75 \mu \mathrm{m}(40 \mathrm{wt} \%)$ and $700 \mu \mathrm{m}(60 \mathrm{wt} \%)$, similar to the A-D blend (Roche et al., 2005, 2006). For this simulation, the bed was $0.25 \mathrm{~m}$ in width and $0.4 \mathrm{~m}$ in height, which gave the same reservoir aspect ratio as in Roche et al. $(2005,2006)$. The resolution of the simulation was chosen to be $5 \mathrm{~mm}$ in both dimensions. Details on the simulation initial and boundary parameters are provided in Table 1. For this simulation the bed was expanded and its solid fraction decreased to 0.57 in order to determine the effect of initial pressurization as the bed compacts. No gas fluidization was used to 
expand the bed, and pore pressure was initially at ambient atmospheric pressure (i.e., pressure-balanced). This simulation was compared with simulations involving a monodisperse grain-size equivalent to the surface mean and volume mean diameters, noted D32 $(160 \mu \mathrm{m})$ and D43 (500 $\mu \mathrm{m})$, respectively.

Another set of 10 simulations involved a bed of $0.25 \mathrm{~m}$ in width by $0.4 \mathrm{~m}$ in height, with the blend of 75 - and $700-\mu \mathrm{m}$ glass beads in proportion from 0 to 1 with 0.1 intervals. In order to show the effect of grain size on the flow shape and dynamics, we calculated the position of the center of mass by numerical integration of the flow mass versus distance at the time where the flow reached creeping motion ( $1.2 \mathrm{~s}$ after release). The center of mass distance was made nondimensional by scaling it to that of the fine monodisperse case $(100 \% 75-\mu \mathrm{m}$ beads). Note that for all simulations we used a particle segregation coefficient of 0.3 (used to replicate particle size segregation) as suggested for bidisperse mixture by Gera et al. (2004). Experiments of Roche et al. (2005, 2006) did not start at constant packing of 0.6 but displayed a Gaussian solid concentration (from concentration $=0.58-0.66)$ centered with its maximum for the blend of $50 \%$ of $75-\mu \mathrm{m}$ beads. Based on their experiments, however, no clear correlation existed between the initial packing of the disperse granular mixture and its runout. Thus, we decided to simulate all dam-break collapse with an initial solid packing of 0.6 , to emphasize the sole effect of size segregation on the flow mobility.

\subsection{Wall Effects}

While the experimental flows are 3-D and momentum is transferred between the flow and side walls, measurements of flow propagation were obtained in 2-D on the sidewalls, and pore pressure measurements were obtained in the center of the channel. In experiments, the variations in flow thickness and runout between the centerline and by the sidewalls are up to $\sim 3 \%$ only, which implies that flat frictional sidewalls had negligible effect on the propagation and emplacement of the experimental flows. Similar conclusions were obtained from work on dam-break collapses of granular flows (Ionescu et al., 2015; Lusso et al., 2017). This is mostly explained by the presence of flat walls allowing the formation of quasi free-slip boundary conditions, where the slip velocity is close to that of the centerline velocity. Additionally, the channel width is $>1,300$ times that of the mean size diameter and the flow thickness at a given location is much smaller than the channel width during most of the flow emplacement. Consequently, in this work we chose to conduct simulations in 2-D, which assumes that we simulate the experimental flows through their centerline.

Studying the effect of wall roughness on the flow kinematics, which may be of importance when dealing with channelized pyroclastic density currents, would require experiments with bumpy walls and may be important to consider in future numerical and experimental studies.

\section{Results}

\subsection{Pressure-Balanced Monodisperse Simulations-KT and Schaeffer Model}

The dam-break collapse of the pressure-balanced granular pile simulated with the KT theory is shown in Figure 1. For comparison, we plot the contours of experimental flows at various timesteps. While we expected the KT approach to have significant limitations at these concentrations, we do include these results so that they can be compared to cases where a frictional stress is added. The KT model predicts a slight (1\%) compaction of the mixture, which results in a pressurization of the gas phase (Figure 1b). The gas pressure reaches $>4,000$ Pa relative to ambient, which equates $>70 \%$ of the bed weight. Consequently, the bed behaves as a viscous gravity current that reaches $1.5 \mathrm{~m}$ in only $0.52 \mathrm{~s}$ after opening of the door. These results do not concur with experiments wherein basal pore pressure of $400 \mathrm{~Pa}$ at most was measured ( 15\% bed weight support), and where the flow did not reach beyond $1.05 \mathrm{~m}$ (see Roche et al., 2010). In such cases (KT theory), the simulated flow is much more mobile than in experiments.

When adding frictional stresses to the kinetic-collisional stresses, as described by the Schaeffer model (Figure 2), the gas pressure reaches values close to that of the KT simulation (Figure 1b). To investigate the role of the elevated pore pressure on the simulated flows shown in Figure 2, we modified the MFIX code so that the permeability of the mixture was 100 times larger (equivalent to a mixture of particles of $750 \mu \mathrm{m}$ ) and did not cause extreme pore pressure while compacting (i.e., the pressure diffusion time scale was very short and the drag force was much lower), but granular stresses were still described with a particle diameter of $75 \mu \mathrm{m}$ (Figure 3). Under these assumptions the pore pressure did not exceed $300 \mathrm{~Pa}$ (Figure 3), more akin to that in the experiments. In summary, the KT simulations show a flow that propagates much faster than in 
a
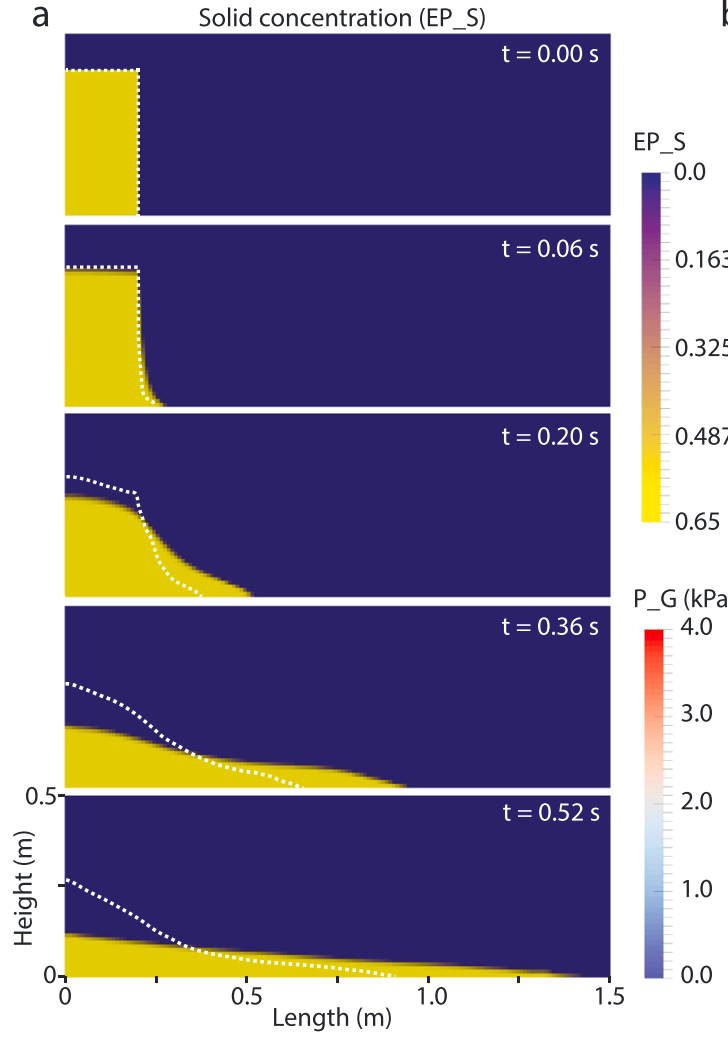

b
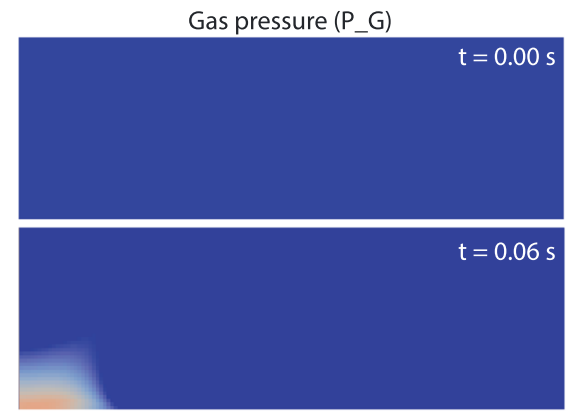

0.487
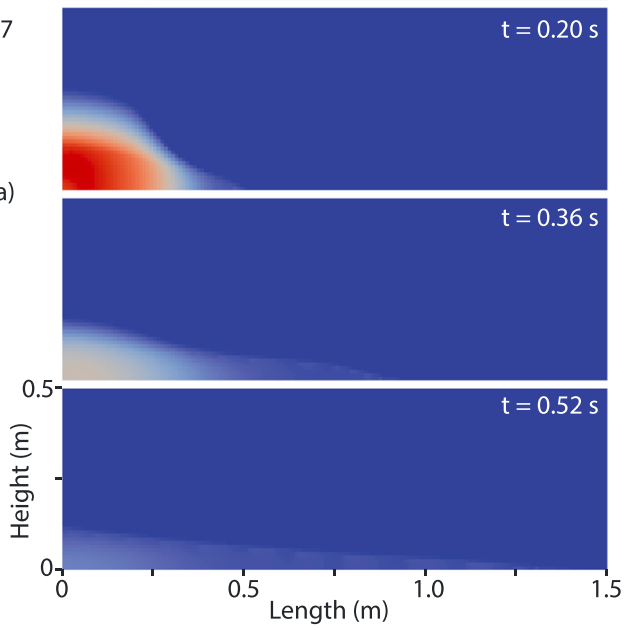

Figure 2. Dam-break collapse simulation of an initially pressure-balanced bed with the Schaeffer frictional model added to the kinetic theory. Solid fractions (a) and gas pore pressure (b) at the various time steps. The white dashed line is the plot of the upper surface of the experimental dense flow (Roche et al., 2010). The vertical scale and horizontal scale indicated in the lowermost figures is the same for all figures above.

experiments (Figure 3a), which reflects the poor description of the near-packing regime by the KT, despite the modification of the RDF at contact. The simulation with the Schaeffer frictional model added to the KT captures the flow dynamics better at all times following the modification of the permeability (Figure 3b) but an artificial (and different from that of experiments) mixture permeability is required to alleviate excess pore pressure to achieve similarity.

\subsection{Pressure-Balanced Monodisperse Simulations-Schaeffer Versus Princeton Frictional Model}

While the Schaeffer model captures roughly the overall flow behavior, it shows that when the flow is close to rest the packing is $\leq 0.64$. However, because the solid pressure across the bed is expected to be depth-variant (ideally hydrostatic in quasi-static regime), such behavior is not fully realistic.

Therefore, we compare the simulations undertaken with the Schaeffer model, wherein we artificially modified the mixture permeability (as described above), and Princeton frictional model (Figure 4). Additionally, both simulations are compared with experiments in terms of flow shape and flow front velocity versus time. In both cases, simulations match experiments relatively well. The Schaeffer model captures better the flow shape in the first $0.06 \mathrm{~s}$, while the Princeton model reproduces better the flow shape at $>0.06 \mathrm{~s}$ as well as the (expected) depth-variance of the packing fraction and the flow front kinematics. The striking difference between both models lies in their description of the solid pressure (Figures $4 \mathrm{c}$ and $4 \mathrm{~d}$ ). The solid pressure plots of the Schaeffer simulation show the rapid formation ( $\sim .2 \mathrm{~s})$ of sharp pressure gradients, which yield formation of a wedge shape in the left corner of the collapsing column and leads to the gently convex shape of the flow near source. In contrast, the Princeton simulations shows a more gradual variation of solid pressure, which leads to the formation of a concave flow surface near source. Note that the shape of the flow where the original column was present $(-0.2$ to $0 \mathrm{~m})$ shows the largest discrepancy between simulations and experiments. 


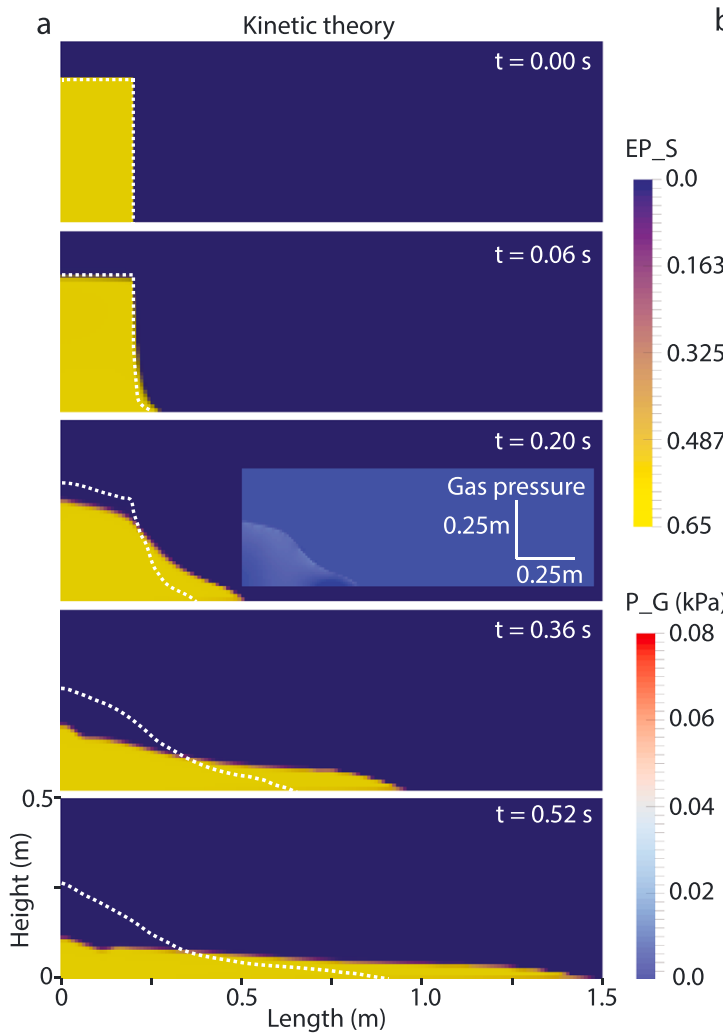

b

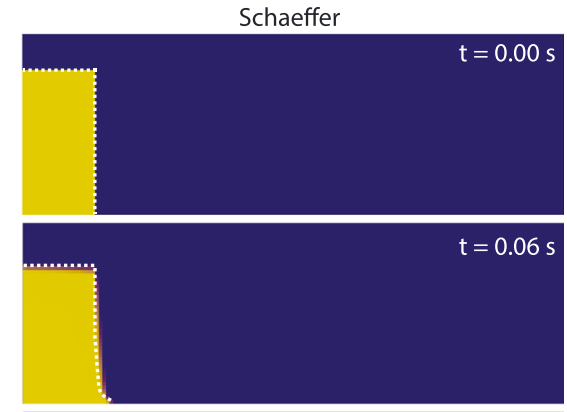

0.487

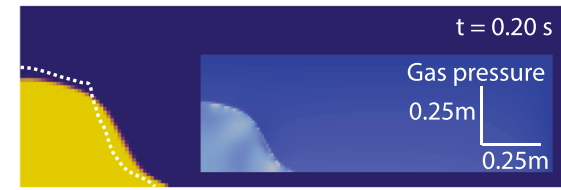

$\mathrm{G}(\mathrm{kPa})$

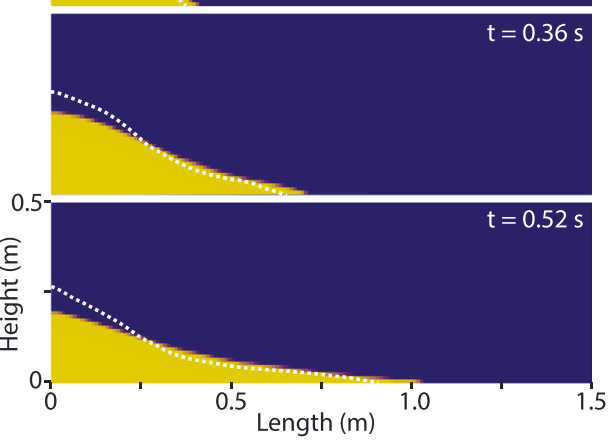

Figure 3. Comparison of the dam-break collapse of pressure-balanced beds after modification of the bed permeability for the same time steps as in Figure 2 for the kinetic theory (a) and Schaeffer models (b). The inserts at $0.20 \mathrm{~s}$ show the gas pore pressure after compaction of the column. Note that $P_{-} G$ was 1 order of magnitude larger in Figures 1 and 2 . The vertical scale and horizontal scale indicated in the lowermost figures is the same for all figures above.

Bearing in mind that the simulation depicting the Schaeffer frictional model has been modified to limit the formation of elevated pore pressure, whereas the simulation using the Princeton frictional model was not impeded by any artificial bed permeability assumptions, the latter model was favored for further investigation.

\subsubsection{Velocity}

We investigated the flow velocity and pore pressure with the Princeton model. The flow structure can be considered as composed of a head, where basal slip occurs, and a body where the no-slip boundary condition is fulfilled for both gas and solid phases. In order to better resolve the velocity of the flow, we doubled the cell resolution (4 times the number of cells; Figures $5 \mathrm{a}-5 \mathrm{c}$ ). In the head, the velocity was quasi linear from base to upper surface (Figure 5c), while it tends to 0 in the base for the body, where the deposit forms, and is linear in the upper flowing portion (Figure $5 \mathrm{~b}$ ). These results are in sufficient good agreement for our purpose. As shown in Figure $5 b$, higher velocities in the head than in the body imply a progressive stretching of the flow over time.

At late stages, when the experimental flow has come to rest, the flow in the model is still in creeping motion with velocities typically $<0.005 \mathrm{~m} / \mathrm{s}$. Therefore, in order to avoid high computational cost, simulations were stopped at $1.3 \mathrm{~s}$ where the flow was in this creeping flow stage, and which was the time at which the experimental flows stopped. The reason the modeled flows did not fully stop is addressed in the discussion.

Another essential comparison between experiments and simulations concerns the flow front kinematics. The plot of the distance versus time of the flow front shows the good match of the simulations with the experiments, not only for the high-resolution run $(0.005 \mathrm{~m})$ but also for the lower-resolution simulation $(0.01 \mathrm{~m}$; Figure $5 \mathrm{~d})$. Additionally, for the high-resolution run, we estimated the nondimensional time as $t /$ 

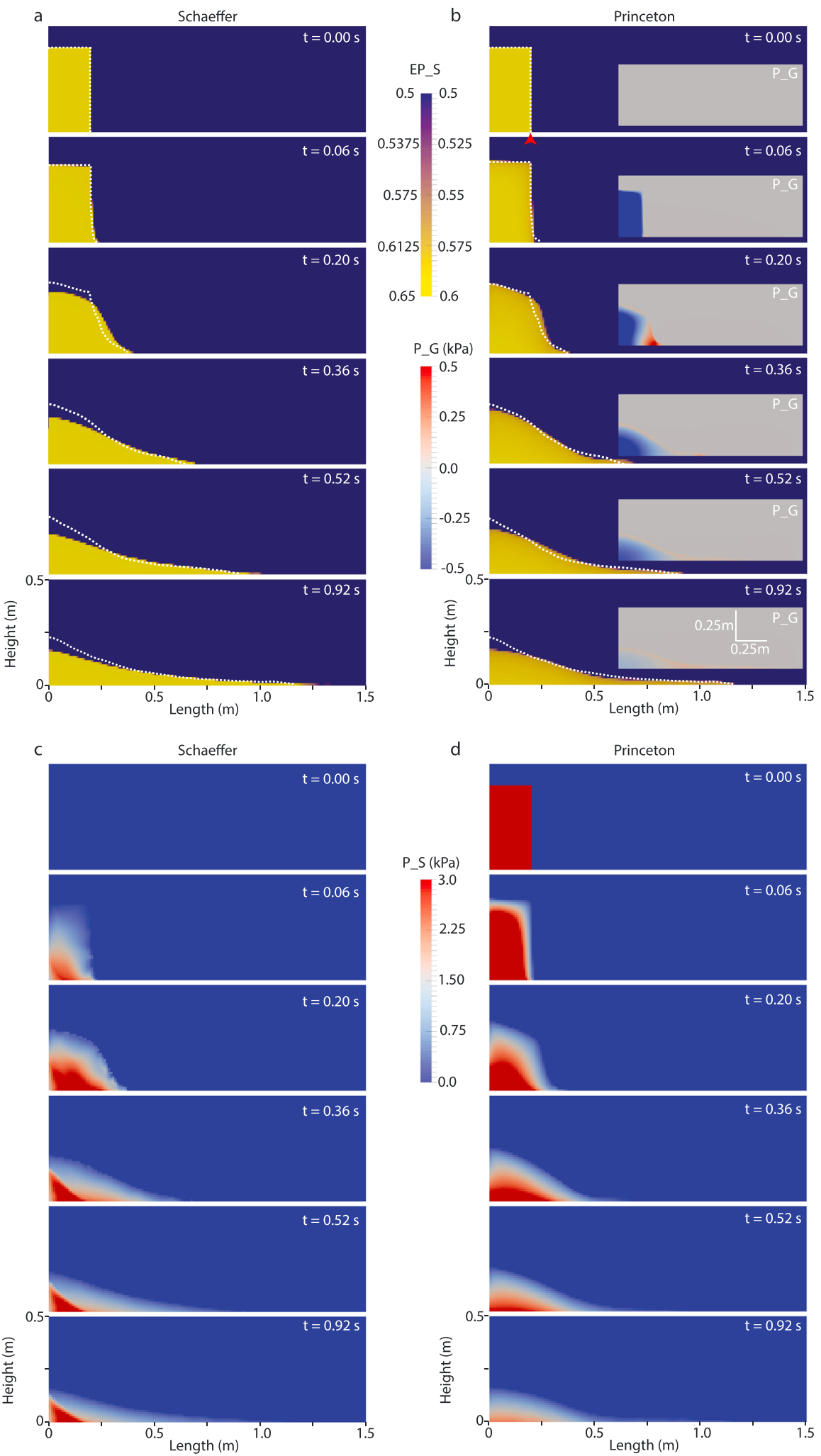

Figure 4. Comparison of simulations of pressure-balanced dam-break collapse using the modified Schaeffer (a and c) and Princeton models (b and d) in terms of solid concentration (EP_S) and solid pressure (P_S). Additionally, the gas pore pressure $\left(P_{-} G\right)$ is plotted as inserts for the Princeton model. The experimental flow contours are also represented as the white dashed line. Note that the simulation with the Schaeffer model used an artificial permeability by using in drag laws a particle size of $750 \mu \mathrm{m}$, ensuring pore pressure to diffuse rapidly 

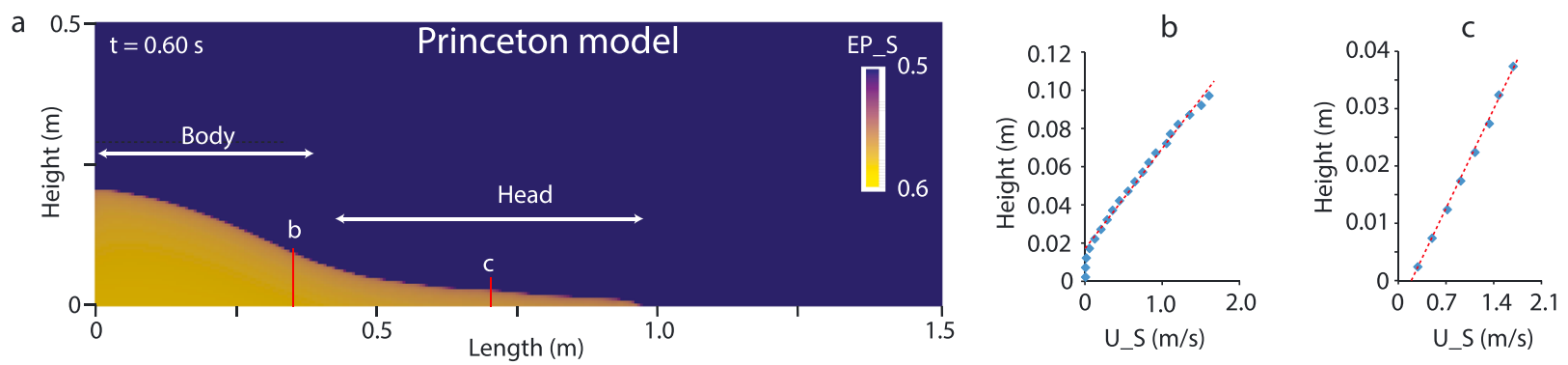

d

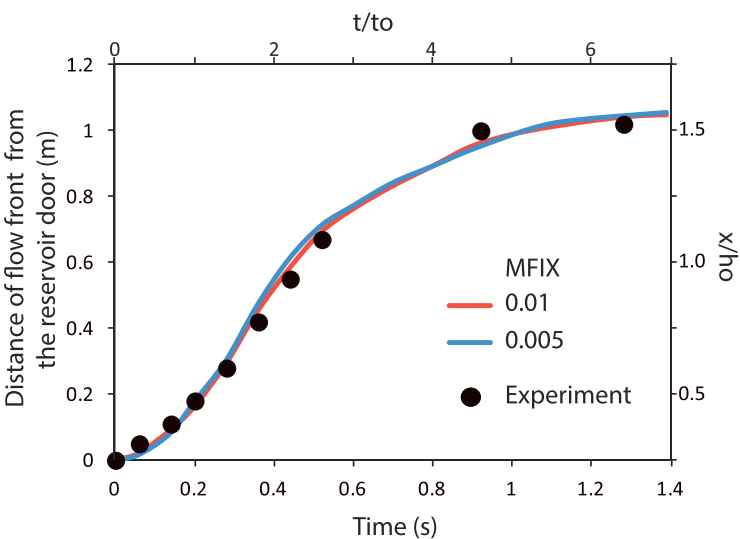

e

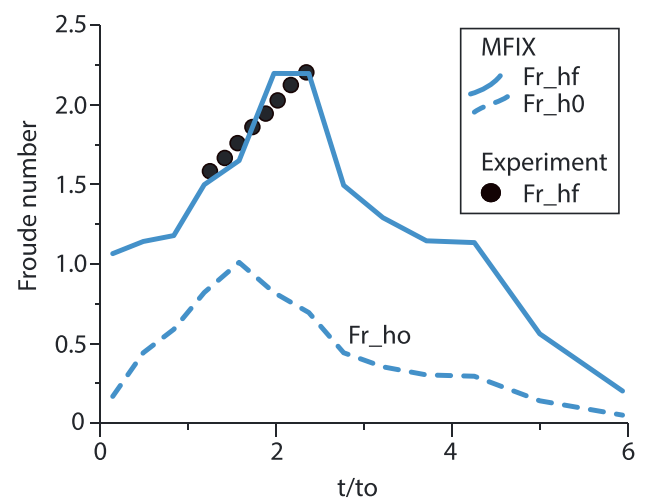

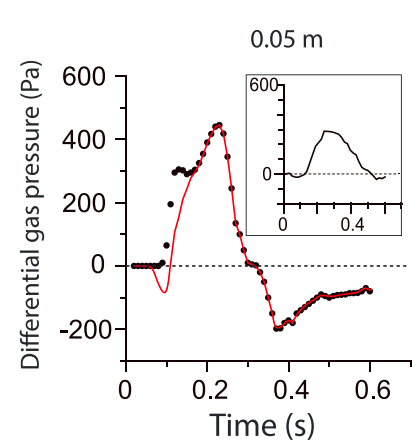

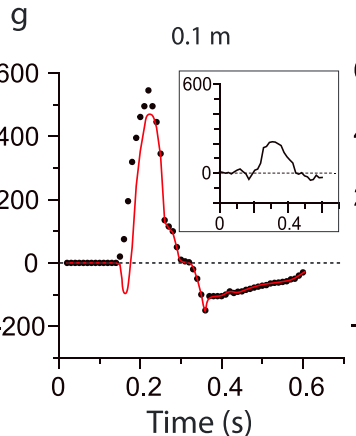

h

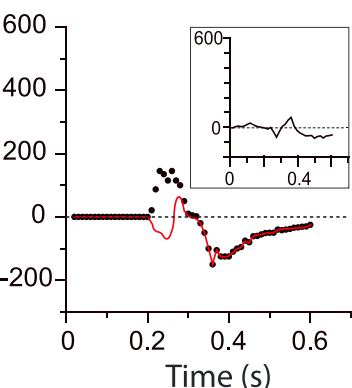

$0.4 \mathrm{~m}$

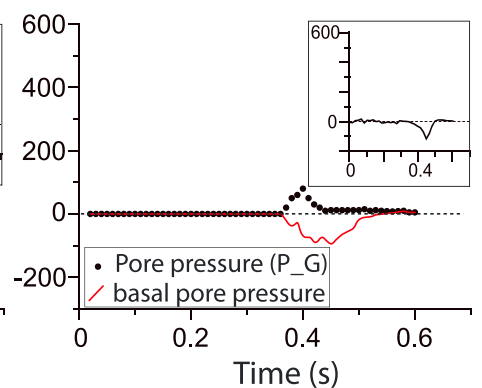

Figure 5. (a) Solid fraction plot and location of the solid velocity profiles in the body (b) and head (c) of the flow simulated with Princeton model. (d) Location of the flow front for simulations at 0.01 and 0.005 grid cell resolution versus experiments (points). (e) Plot of the Froude numbers $F r_{h 0}$ and $F r_{h f}$ as a function of nondimensional time $t / t_{0}$, where $t_{0}=\left(h_{0} / \mathrm{g}\right)^{0.5}$ with $h_{0}$ as the bed height at $t=0.0 \mathrm{~s}$. The black points are from experimental measurements provided by Roche et al. (2008). (f-i) The pore pressure $P^{-} G$ (black points) versus the synthetic basal pore pressure signal (red line, calculated from equation (33)) is shown for the simulation of pressure-balanced dam-break collapse at 0.05 (f), 0.1 (g), $0.2(\mathrm{~h})$, and $0.4 \mathrm{~m}$ (i). Inserts of plots in (f)-(i) are the experimental basal pore pressure data $\left(P_{\text {basal }}\right)$ from Roche et al. (2010), where the scales are the same as for the larger plots ( -100 to $600 \mathrm{~Pa}$ and 0 to $\left.0.6 \mathrm{~s}\right)$.

$t_{0}$, where $t_{0}=\left(h_{0} / \mathrm{g}\right)^{0.5}$ and the nondimensional position of the flow front as $\mathrm{x} / h_{0}$ (Figure $5 \mathrm{~d}$ ). Further investigation of the flow front dynamics was undertaken by calculating the Froude numbers noted $F r_{h 0}$ (initial Froude number) and $F r_{h f}$ (flow Froude number) as

$$
F r_{h f}=\frac{U_{f}}{\left(g h_{f}\right)^{0.5}} \text { and } F r_{h 0}=\frac{U_{f}}{\left(g h_{0}\right)^{0.5}}
$$

where $h_{f}$ was the mean flow height, which was taken as the average height of the central third of the flow (from $0 \mathrm{~m}$ onward) for consistency with Roche et al. (2008), and $h_{0}$ was the height of the column in the reservoir. 
The $F r_{h 0}$ rose from 0.2 to 1 from $t / t_{0}$ of 0.03 to 1.8 and declined down to 0 at $t / t_{0}=6$. Subsequently, the flow Froude number $\mathrm{Fr}_{h f}$ gave values of 1.15 at $t / t_{0}=0.03$, peaked up to 2.25 at $t / t_{0}=2.5$, and declined afterward. Measurements of the $F r_{h 0}$ and $F r_{h f}$ provided by Roche et al. (2008) are in agreement with these simulations. 3.2.2. Pore Pressure

Intuitively, one would expect pore pressure to negligibly affect the initially pressure-balanced flow. In fact, dense granular dam-break collapse are generally treated with incompressible algorithms (Lagrée et al., 2011), but this can lead to ill-posedness of the equations at low and high inertial numbers (Barker et al., 2015). However, here we treat the flow as compressible (i.e, concentration changes are captured), with a compressible fluid phase, so that it can experience compression and dilation. Concentration changes impose pore pressure variations across the flow, which are sustained for hundreds of milliseconds (Figure 4) due to a low mixture permeability of $\sim 1.0 \times 10^{-11} \mathrm{~m}^{2}$.

Experiments showed that basal pore pressure measured for initially pressure-balanced flows was not solely positive but also negative with respect to ambient depending on the position of the flow relative to the sensor (Roche, 2012; Roche et al., 2010). In a time series of experimental bed pressures, a negative trough preceded a positive pressure excursion, which was itself followed by a second negative trough of longer duration. In simulations, the basal pore pressure is calculated across the flow. The meaning of the negative pore pressure in experiments was thought to be related to basal slip boundary condition as shown in Roche et al. (2013), and the maximum value of the negative peak was empirically correlated to the flow front velocity. This empirical law was used to approximate front velocities of natural flows (see Roche et al., 2016). Here we show that the whole pore pressure signal can be calculated based upon local physical properties of the flow.

A pressure sensor in experiments measured the differential pressure between ambient atmospheric pressure below the diaphragm and pressure generated by the flow above. The basal pore pressure $P_{\text {basal }}$ can be theoretically expressed as

$$
P_{\text {basal }}=P_{-} G-P_{\text {dynamic }}=P_{-} G-\frac{1}{2} \rho_{b} v_{\text {slip }}^{2}
$$

where $P_{-} G$ is the pore pressure, $\rho_{b}$ is the mixture density at the base, and $v_{\text {slip }}$ is the mixture velocity at the base. If the mixture was static and fluidized (i.e., Umb) then $P_{-} G=g \int_{0}^{H} \rho N \mathrm{~d} z$, where $H$ was the mixture height, $N$ was the degree of bed support ( $=1$ when fully fluidized), and $\rho$ was the mixture density.

In simulations, the pore pressure $P_{-} G$, mixture density and velocity at the base are calculated by solving the momentum equations of the gas and solid phases, thus providing the parameters required to estimate the synthetic basal pore pressure (equation (33)) shown in Figures 5f-5i. Both the $P_{\text {basal }}$ and $P_{-} G$ total pore pressure of the gas are plotted at four distances from the gate: 0.05 (Figure 5f), 0.1 (Figure $5 \mathrm{~g}$ ), 0.2 (Figure 5h), and $0.4 \mathrm{~m}$ (Figure 5i), thus showing the evolution of the flow. At all times and all distances, the pore pressure signal depicts a negative peak, followed by a positive peak and preceding another negative peak. The basal pore pressure that takes into account the dynamic term of the pressure is locally influenced by the slip and shows that basal slip of the head modifies the pressure signal to an extent where the synthetic basal pore pressure becomes negative (with respect to atmosphere). The merging of the $P_{\text {basal }}$ and $P_{-} G$ data signifies that the dynamic pressure term becomes negligible due to the slip velocity that tends to 0 . In summary, the first negative peak in the basal pressure data $\left(P_{\text {basal }}\right)$ is a direct function of the basal slip velocity as shown in equation (33) and solely forms when the dynamic pressure is larger than the total pressure (see equation (33)).

With distance, the respective proportion of the first negative to positive peaks grows, due to the static pressure diffusion. Additionally, the second negative peak reflects the actual static pressure of the mixture and is related to internal shear gradients (Lube et al., 2019), where dilation leads to negative pore pressures. These results illustrate the complexity of understanding the pore pressure data without other measurements such as local slip, mixture density, and degree of bed support.

Due to positive pore pressure, the bed weight appears to be supported up to 15-20\% through pore fluid drag. Note that maximum basal pore pressure values predicted by our simulation (up to 400-460 Pa) are slightly larger than measured in experiments (up to $350 \mathrm{~Pa}$ ) but extreme values of both negative pressures $(-100$ to $-150 \mathrm{~Pa}$ ) are in good agreements with experiments (Roche et al., 2010). 


\subsection{Dam-Break Collapse of a Fully Fluidized Bed}

\subsubsection{Flow Shape and Solid and Gas Pressure}

In this set of three simulations, we used the setup explained in section 2 and shown in Figure 1a. It is similar to that of the pressure-balanced case with the essential exception that the bed is initially fluidized and expanded by $1.6 \%, 3.3 \%$, or $5.2 \%$.

Figures $6 \mathrm{a}$ and $6 \mathrm{~b}$ show the evolution of the numerical flow and the contours of the experimental flow. The solid pressure is lower than in the pressure-balanced counterpart (Figure 4 ) by nearly 1 order of magnitude ( $t$ $=0.08 \mathrm{~s}$, Figure $6 \mathrm{a}$ ), which strongly reduces the frictional stresses. This is due to a lower packing fraction (by $\sim 2 \%$ ) and higher pore pressure ( $>10$ times). Interestingly, when the bed is fully fluidized, the solid pressure does not go to 0 (Figure $6 \mathrm{a}, t=0.0 \mathrm{~s}$ ), whereas the bed weight is $94 \%$ supported as shown in the gas pressure profile where basal pressure $=5.5 \mathrm{KPa}$.

As the bed collapses, its dilation yields low gas pore pressure in the bed ( $t=0.08 \mathrm{~s}$, Figure $6 \mathrm{~b}$ ). Later, the bed compacts and high pore pressure forms, and subsequently slowly diffuses in the following second of simulation.

Compared to experiments, the shape of the flow is relatively well captured initially but differs in late stages $(t$ $=0.92 \mathrm{~s}$ ), as the flow pile heights shows a larger peak at $0.3 \mathrm{~m}$ and is significantly thinner from $1 \mathrm{~m}$ onward. Overall, the shape of the deposit in experiments, which shows a low point from 0 to $0.25 \mathrm{~m}$, is expected to be due to further fluidization of the column after opening of the door as the gas flux was not turned off.

\subsubsection{Flow Front Velocity}

The flow front velocity is shown in Figure $6 \mathrm{c}$. Three stages are shown: (i) the collapse phase, for $t / t_{0}<1.5-2$, (ii) the constant velocity phase that lasts until $t / t_{0} \sim 3.5$, and (iii) the stopping phase $\left(t / t_{0}>\sim 3.5\right)$. In the collapse phase the flow front velocity increases, while the $F r_{h f}$ decreases to about 2 and Fr_ho increases up to values close to 1.2 (Figure $6 \mathrm{~d}$ ). This reflects the increase of the flow front height. In the constant velocity phase the $\mathrm{Fr}$ _ho is close to $1.4 \approx 2^{0.5}$. This behavior is typical of inviscid fluid gravity currents (Britter \& Linden, 1980; Marino et al., 2005; Shin et al., 2004). In the stopping phase the flow front velocity wanes and both Fr_ho and $F r_{h f}$ decrease. Note than in simulations, the flow front dilutes and does not stop at $1.28 \mathrm{~s}$ as in experiments.

Additionally, we investigated the sensitivity of the simulation to the initial solid packing fraction of the bed and show that for initial concentration of 0.57 the flow is faster than in experiments and reaches final experimental runout of $\sim 2.2 \mathrm{~m}$ in $1.0 \mathrm{~s}$. When the initial bed concentration is 0.59 , the flow front is slower than in experiments. While the flow packing fraction differs by only $0.1-0.2 \%$ at most for the $0.57-0.59$ initial concentration simulations, the role of initial dilation and contraction is significantly different. The flow experiences no contraction but a dilation of $0.1 \%$ in the 0.59 case, while it experiences a compaction of $1.7 \%$ in the 0.57 case. Because gas is compressible and the mixture permeability is low, the gas pressure between these two cases after $0.08 \mathrm{~s}$ can be different by $1 \mathrm{kPa}$. Furthermore, the solid pressure nearly doubled in the 0.59 case with respect to the 0.57 case.

\subsubsection{Basal Pore Pressure}

Similar to section 3.2.2, we estimated from the pore pressure $\left(P_{-} G\right)$, slip velocity, and flow density the synthetic basal pore pressure at various distances in numerical simulations. The gas pore pressure (Figure 6e) of $5.5 \mathrm{kPa}$ at initial stage indicates a bed support of $\sim 100 \%$, which corresponds to the onset of bubbling in experiments at gas velocities noted "Umb" (Roche et al., 2010). After opening the reservoir gate $(t=0 \mathrm{~s})$, the pressure at the base of the reservoir (distance $=-0.125 \mathrm{~m}$, Figure $6 \mathrm{e}$ ) declines rapidly to $1 \mathrm{kPa}$ and waxes while oscillating around values of $4 \mathrm{kPa} . P_{-} G$ further declines exponentially from $0.25 \mathrm{~s}$ onward with little fluctuations. These fluctuations coincide with bed dilation (when flow pressure is declining) and contraction (when pressure increases). The compressible behavior of the bed (change in packing fraction) explains the same observations made in the basal pore pressure by Roche et al. (2010). Note that due to negligible slip at the base, the gas pore pressure $P_{-} G$ and basal pore pressure curves overlap at all times.

At $0.05 \mathrm{~m}$ from the door (Figure 6f), the $P_{-} G$ is positive during the simulation duration, whereas the basal pore pressure signal shows a small $(-0.1 \mathrm{kPa})$ low peak prior to a much large $(3.5 \mathrm{kPa})$ positive peak. The minima are concurrent with significant slip that lasts until $0.3 \mathrm{~s}$, indicated as both $P \_G$ and basal pore pressure data merge. 

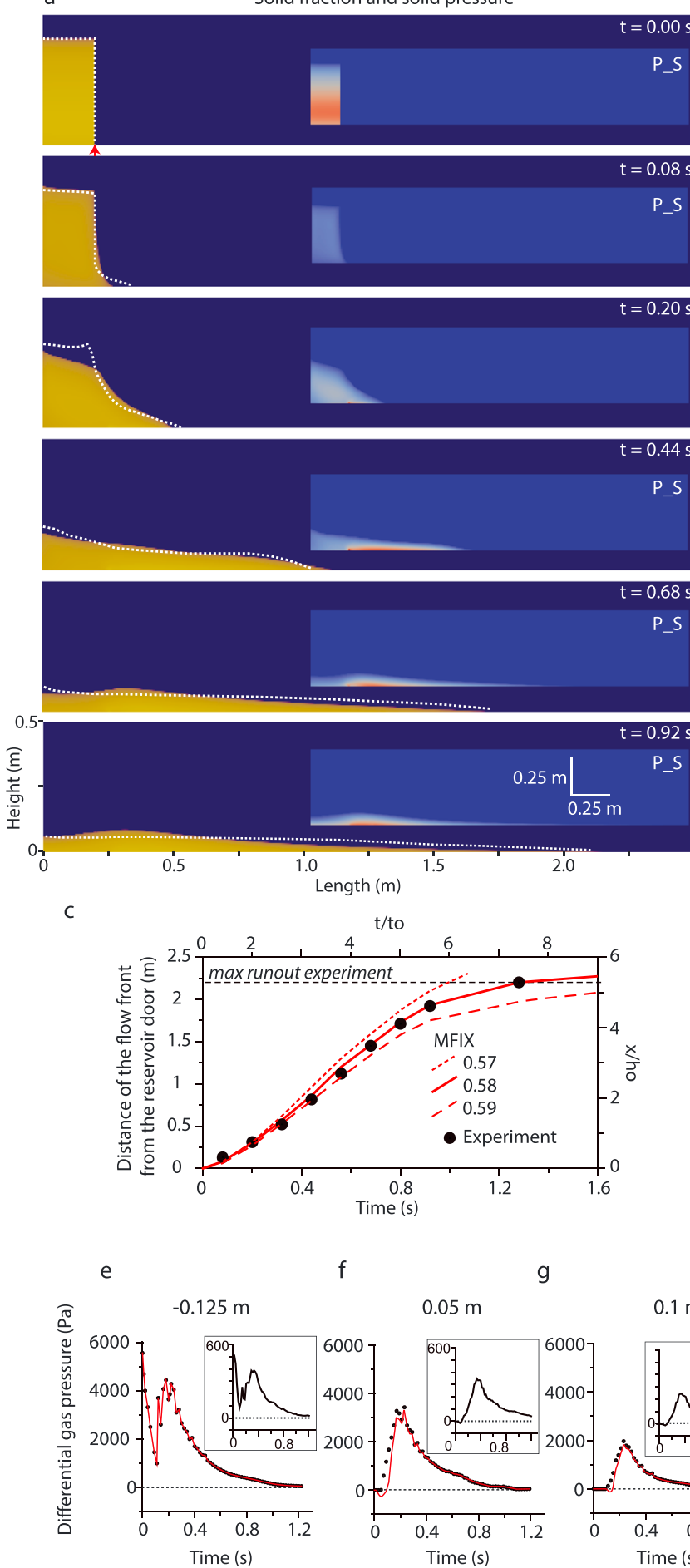

f

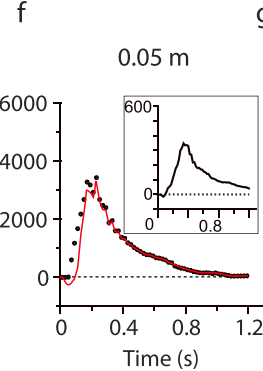

g b

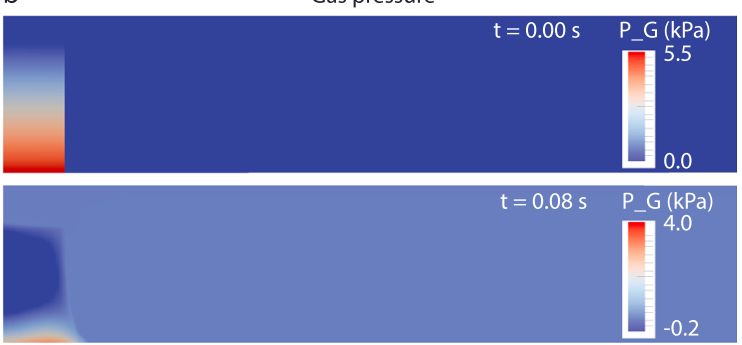

$-0.575$

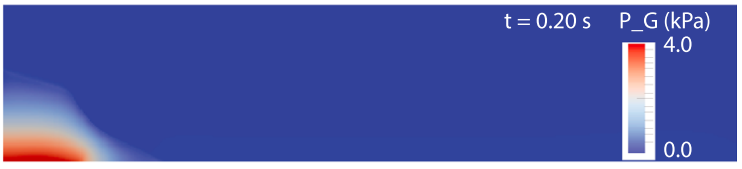

$\mathrm{P}_{-} \mathrm{S}(\mathrm{kPa})$
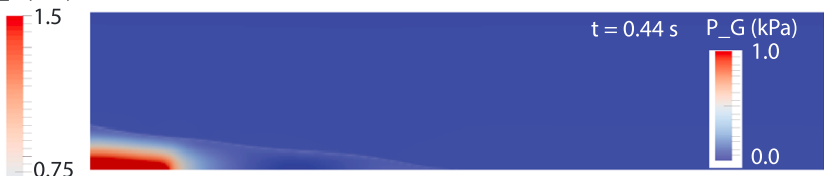

0.75



0.0
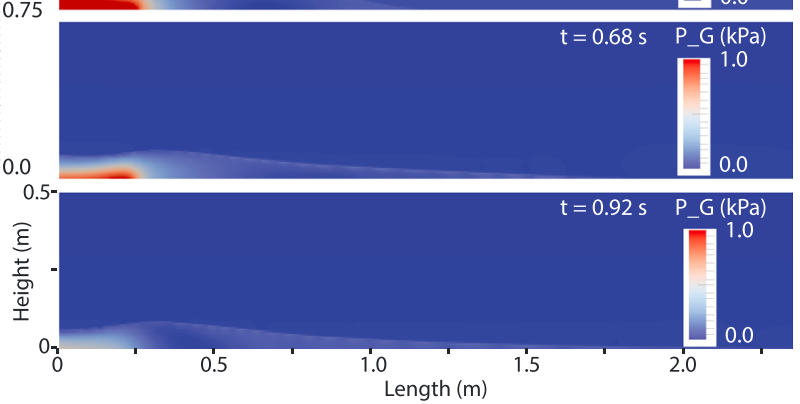

d

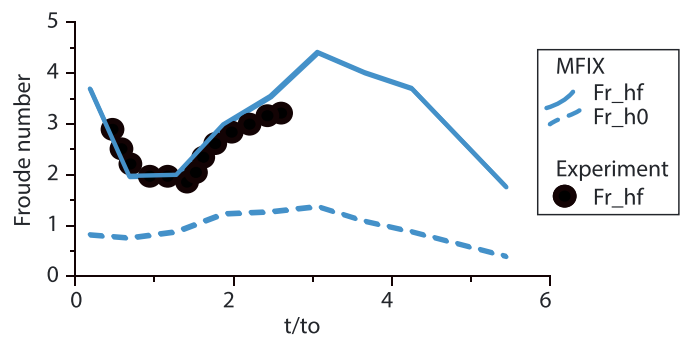

h

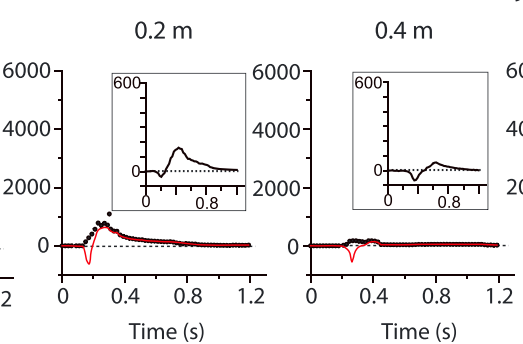

j

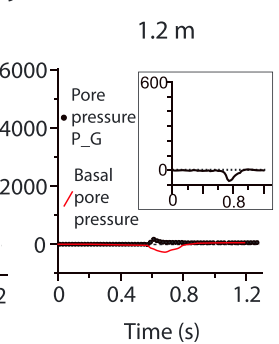

Figure 6. Results of the initially fluidized simulation (at the onset of bubbling in Roche et al., 2008). (a) Plots of solid fractions (dashed line is the flow contour in experiments) with inset of the solid pressure (P_S). (b) Same as (a) but the gas pore pressure $\left(P \_G\right)$ is shown instead. (c) distance of the simulated flow front (red line) versus time against experiments (black points). The two dotted lines represent the results of two additional simulations where the initial solid concentration was 0.57 (red dotted lines) and 0.59 (red dashed line). (d) Plot of the Froude number $F r_{h 0}$ and $F r_{h f}$ as a function of nondimensional time $t / t_{0}$, where $t_{0}=\left(h_{0} / \mathrm{g}\right)^{0.5}$ with $h_{0}$ as the bed height at $t=0.0 \mathrm{~s}$. The black points are from experimental measurements provided by Roche et al. (2008). Static and basal pore pressure in the fluidized simulation at $-0.125(\mathrm{e}), 0.05(\mathrm{f}), 0.1(\mathrm{~g}), 0.2(\mathrm{~h}), 0.4(\mathrm{i})$, and $1.2 \mathrm{~m}(\mathrm{j})$. Basal pore pressure is calculated from local slip, flow density, and pore pressure $P^{-} G$ as described in equation (33). Inserts of plots in (e)-(j) are the experimental basal pore pressure data $\left(P_{\text {basal }}\right)$ from Roche et al. (2010), wherein the scales are equivalent to that of the larger plots $(-1,000$ to $6,000 \mathrm{~Pa}$, and 0 to $1.3 \mathrm{~s})$. The vertical scale and horizontal scale indicated in the lowermost figures is the same for all figures above. 
Both pressure data at 0.1 (Figure 6g), 0.2 (Figure 6h), and $0.4 \mathrm{~m}$ (Figure 6i) show similar trend to that of 0.05 $\mathrm{m}$. Meanwhile, the negative pressure peak preceding the positive peak increases its proportion from $10 \%$ to $140 \%$, explained by the increasing contribution of the dynamic pressure in the $P_{-} G$ measured. Similar to the approach of Roche et al. (2013), the minimum value of the first negative peak $(\Delta \mathrm{P})$ can be empirically correlated to the flow front velocity $\mathrm{U}_{\mathrm{f}}$. We find that $P=0.072 \rho U_{f}^{2}$, which is in good agreement with $P \sim 0.060 \rho U_{f}^{2}$ found empirically by Roche et al. (2013).

With time, the pore pressure diffuses, thus lowering $P_{-} G$ as the flow stretches and subsequently thins. At 1.2 $\mathrm{m}$ (Figure $6 \mathrm{j}$ ), the basal pore pressure measured resembles that of a (initially) pressure-balanced flow (see Figures 5f-5i), as the signal shows mainly a negative peak caused by the sliding head, which causes $P_{\text {dynamic }}>P_{-} G$ (thus, $P_{\text {basal }}<0$ ). However, the second negative pressure peak following the positive peak in the basal pore pressure data shown by the pressure-balances cases does not form in fluidized simulations. All these results are in very good agreement with the basal pore pressure data measured in experiments (Roche et al., 2010). In particular, the simulation shows that the pore pressure in the head $\left(P \_G\right)$ is larger than atmospheric pressure whereas the basal pore pressure may be below ambient.

\section{Discussion}

\subsection{Frictional Models}

As generally understood, the original KT (Lun et al., 1984) developed for frictionless particles in dilute flows is not suited to describe flows of solid fraction exceeding $\sim 0.4$, and even with modifications of the energy dissipation rate, for instance as proposed by Jenkins and co-workers (Jenkins \& Berzi, 2010; Jenkins \& Zhang, 2002; Stefan, 2009), the approach cannot capture flow frictional stresses in the dense regime for transient flows. Presently, the best way to account for the complex dynamics of dense flows is to add a frictional model to the KT, and this helps to better describe dense flows in steady bin discharge, for instance (Benyahia, 2008). The validation study of Benyahia (2008) showed that the Princeton frictional model showed better agreement against DEM simulations. Our transient flow simulations lead to a similar conclusion. While future comparison studies between volcanic large-scale experiments and MFIX simulations are needed, the present study suggests that the Princeton (Srivastava and Sundaresan) frictional model should be preferred to the Schaeffer model in order to describe dense frictional flows in concentrated gravity currents such as those often encountered in the basal regions of pyroclastic density currents.

\subsection{Stopping Criteria and Creeping Granular Media}

The largest discrepancy between experiments and simulations arise in the quasi-static regime and in the creeping motion of the flow in its final stages. We showed that while the flow front kinematics matched experiments for almost all the flow emplacement, the front did not stop at the maximum distance observed in experiments but was reduced to a small $(<0.005 \mathrm{~m} / \mathrm{s})$ creeping velocity. There are two reasons for this behavior. The first is numerical, and the second is due to the rheophysics involved. The coarseness of the grid leads to dilution of the flow near its interface and in particular at the front. As described in section 2 (Methods), the flow rheology is highly dependent on the local particle concentration. Here, because the flow front boundary does not systematically overlap with a cell boundary, it dilutes and therefore acquires a lower viscosity. This effect could be minimized by increasing the resolution in these regions (such as with adaptive gridding) or using numerical methods that better capture these concentration gradients. For example, models that treat the granular collapse by neglecting the gas phase often use other types of grids that deform: (1) the Material Point Method (Dunatunga \& Kamrin, 2015), where information such as momentum, mass and stress are stored on material points that move as the flow advances; (2) the regularization method implemented with Taylor-Hood finite elements for space discretization and an implicit Euler scheme with linearization for time discretization, which uses an Arbitrary Lagrangian Eulerian to deal with the displacement of the domain (Lusso et al., 2017).

The second reason behind the nonstopping of the flow is related to the physical description of the frictional stresses. By definition, the Princeton model cannot have the term $S_{s}: S_{s}+\frac{\theta_{s}}{d^{2}}=0$, thus preventing the model from describing a flow that would strictly stop, although flows can have effective viscosities such that they move exceedingly slow. 
Strictly speaking, this means that deposition is not fully resolved, although for practical purposes if the creeping motion decays significantly below the velocities of the flow (often 3-4 orders of magnitude smaller), the very slowly creeping bed provides a reasonable approximation to the deposit. In other models, deposition is generally approximated (but not often really defined) when the flow reaches creeping (Lagrée et al., 2011). To formalize this, deposition can be approximated by using the inertial number. In Figures $7 \mathrm{a}$ and $7 \mathrm{~b}$ we show plots of the inertial number across the flow (equation (3) where the shear rate is replaced by the second invariant of the deviator of the strain rate tensor $|D|$; Jop et al., 2006). We use this parameter for two reasons: (1) at low inertial number $\left(I<\sim 10^{-1}\right)$, granular flows become less frictional than when in the inertial regime $\left(I>\sim 10^{-1}\right)$, and the inertial number reaches its lowest value when quasi static $\left(I<\sim 10^{-3}\right)$, and (2) it emphasizes the role of pressure and shear. Within a fluidized bed, the solid pressure is much lower than the initially pressure-balanced counterpart so that deposition is delayed, and low shear would be associated with the stopping motion of the flow. For these reasons, one could use the quasi-static regime with $I<\sim 10^{-3}$ as a proxy for the deposit. This approach could be valuable when dealing with simulations of natural geophysical flows (e.g., pyroclatic currents (Benage et al., 2016; Dufek et al., 2009; Dufek \& Bergantz, 2007)), provided that the computational grid resolution does not impede the formation of a dense (near packing) basal bedload or flow.

\subsection{Pore Pressure}

While typically dam-break granular flow simulations do not treat the fluid phase when dealing with low viscosity fluid (i.e., air; Staron et al., 2014) because the particle size is too coarse to allow elevated pore pressure to form, the present simulations involving fine particles show the formation of negative and positive pore pressure and its evolution. Note that in small-scale pressure-balanced experiments, particle size $<100$ $\mu \mathrm{m}$ typically does not allow elevated pore pressure to form. The characteristic stresses acting on a particle are the static stress $\sigma_{n}$, viscous stress $\sigma_{v}$ and the inertial stress $\sigma_{i}$. In order to assess the role of the fluid on particle through viscous stresses, one can define characteristic times as (Amarsid et al., 2017; Cassar et al., 2005; Courrech du Pont et al., 2003):

$$
\begin{gathered}
t_{s}=d\left(\frac{\rho_{s}}{\sigma_{n}}\right) \\
t_{v}=d\left(\frac{\rho_{s}}{\eta_{\text {fluid }} \dot{\gamma}}\right)^{0.5} \\
t_{i}=\frac{1}{\dot{\gamma}}
\end{gathered}
$$

with $t_{s}, t_{v}$ and $t_{i}$ as the static, viscous and inertial characteristic times, respectively.

and their ratios gives the nondimensional numbers $I$ and $J$.

$$
\begin{gathered}
I=\frac{t_{s}}{t_{i}}=\left(\frac{\sigma_{i}}{\sigma_{n}}\right)^{2} \\
J=\frac{t_{s}}{t_{v}}=\sqrt{\frac{\sigma_{v}}{\sigma_{n}}}=\sqrt{\frac{\eta_{\text {fluid }}}{\sigma_{n}}}
\end{gathered}
$$

The Stokes number is a ratio of viscous over inertial characteristic times:

$$
S t=\left(\frac{t_{v}}{t_{i}}\right)^{2}=\frac{\sigma_{i}}{\sigma_{v}}=\frac{I^{2}}{J^{2}}=\frac{\rho_{s} d^{2} \dot{\gamma}}{\eta_{\text {fluid }}}
$$

where $\eta_{\text {fluid }}$ is the gas viscosity chosen as that of air. In simulations, we estimate the Stokes number to be between $10^{-5}-10^{2}$, where St $<1$ (Boyer et al., 2011) means that flow span the "inertial" and "viscous" regimes, where in the latter viscous forces become nonnegligible.

Pore pressure can have a significant effect on the flow dynamics. For instance, in water-particle flows such as debris flows, pore pressure is involved into a feedback where dilation and compaction lead to pore fluid 
a

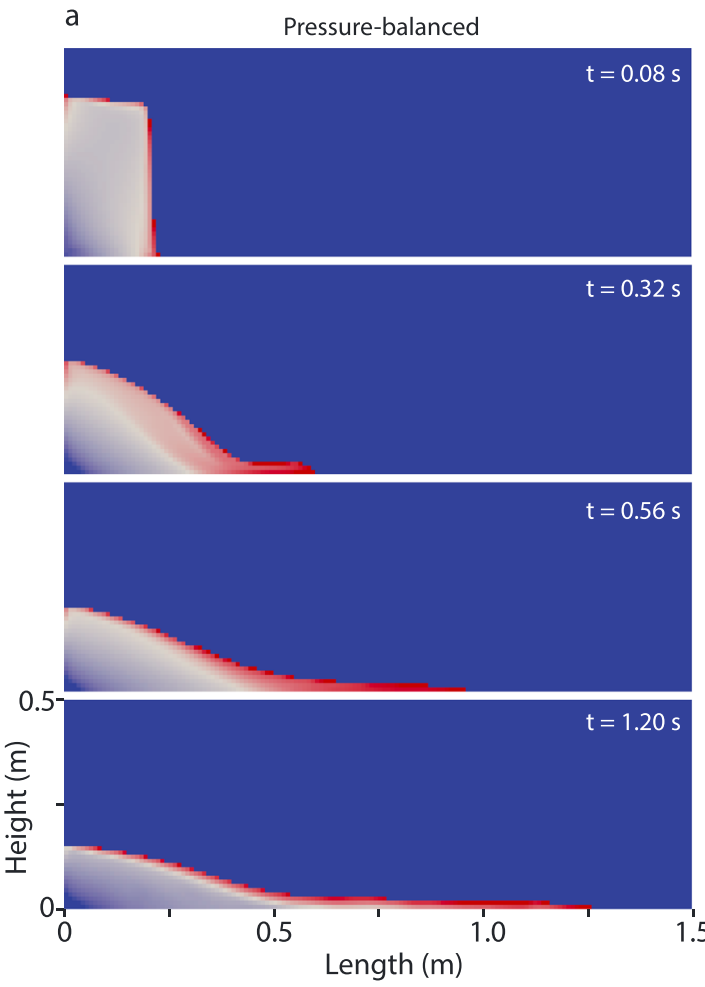

C

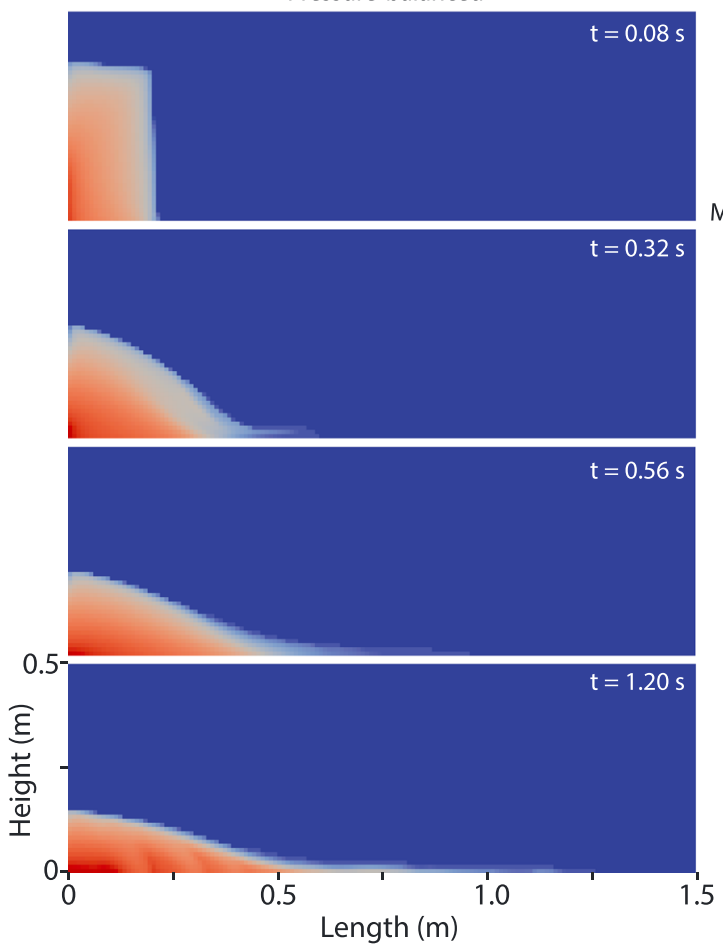

b

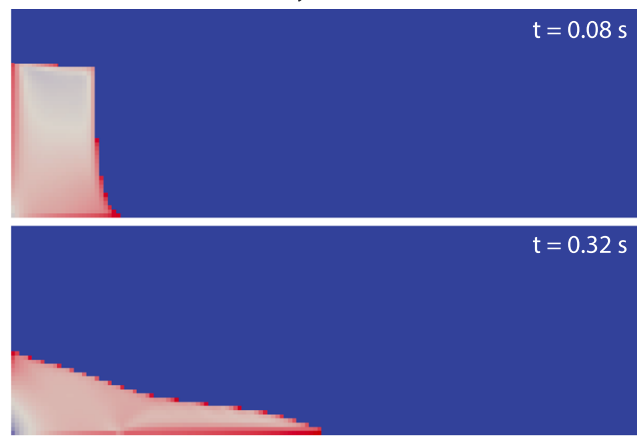

$\mathrm{t}=0.56 \mathrm{~s}$

$10^{-6}$

$10^{-8}$

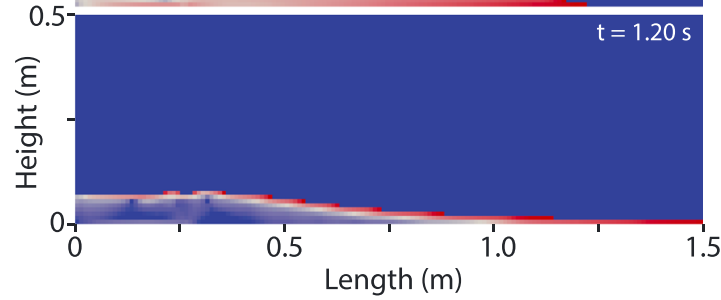

d

Initially fluidized

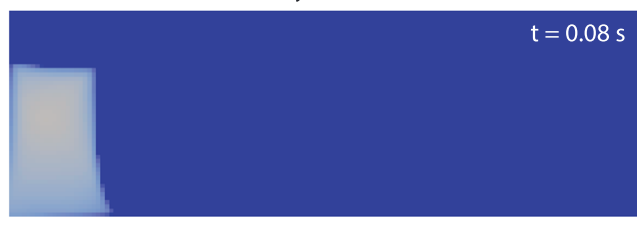

$\mathrm{t}=0.32 \mathrm{~s}$

500
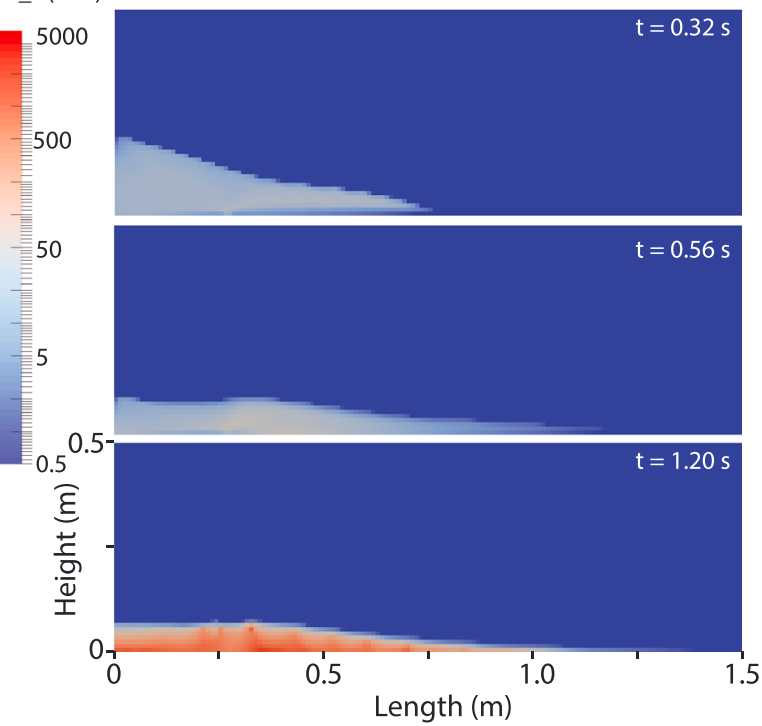

Figure 7. Inertial number (equation (3)) plot for pressure-balanced (a) and fluidized bed (b) simulations with 0.01-m resolution. Plot of the solid viscosity MU_S (equation (29)) for the pressure-balanced (c) and fluidized (d) cases at similar time steps as in (a) and (b). The vertical scale and horizontal scale indicated in the lowermost figures is the same for all figures above. 
pressure decrease and increase respectively (Iverson, 2005). Phenomenologically, the shear stress in debris flows can be defined as a function of the friction coefficient as:

$$
\tau=\cos (\alpha)\left(\sigma-P_{G}\right) \mu_{s}=\cos (\alpha) \sigma(1-N) \mu_{s}
$$

(Iverson, 1997), where $P_{G}$ is the pore fluid pressure, $\alpha$ is the slope angle, $\sigma$ is the normal stress, and $\mathrm{N}$ is the fractional normal stress support. A similar approach has been used to simulate dense gas-particle flows with a depth-average approach (Gueugneau et al., 2017) or to predict the flow runout (Breard et al., 2017). The product $(1-N) \mu_{s}$ is often referred to as the effective friction coefficient, and can take very small values as $\mathrm{N}$ increases. However, equation (40) predicts that at full bed support the shear stress is equal to 0 as $N=$ 1. This behavior is not realistic because the normal stress or solid pressure $\left(\mathrm{P} \_S\right)$ persists $\left(\mathrm{P} \_\mathrm{S} \gg 0\right)$ in fully fluidized cases (at column base it is of order of $10^{2} \mathrm{~Pa}$, Figure $6 \mathrm{~b}$ ). When the bed is fully fluidized, particles still interact with each other so that a solid pressure persists in the bed though the bed weight is supported by fluid drag. This is best illustrated through plots of the solid viscosity (equation (29)) for pressure-balanced and fluidized bed flows (Figures 7c and 7d). The mean solid viscosity of the fluidized bed is about 1 to 2 orders of magnitude lower initially than the pressure-balanced counterpart but remains larger than 1 Pa $\mathrm{s}$, except near the final stage where granular stresses are equivalent to that of the pressure-balanced flows due to the complete pore pressure dissipation. A comparison of the inertial number and viscosity plots shows that high viscosity is concurrent with low inertial number, as the inertial number is positively correlated to the shear rate (Figures $7 \mathrm{a}-7 \mathrm{~d}$ ).

When looking at the effect of pore pressure on flow viscosity, one notices that the granular viscosity is a function of the solid pressure (equation (41)).

$$
\eta_{\text {granular }}=\frac{\mu(I) P}{|D|}
$$

where $P$ is the confining solid pressure and $|D|$ is the second invariant of the deviator of the strain rate tensor. The solid pressure decreases due to (1) bed expansion through drag as the gas advects through the permeable mixture, (2) partial bed support, which lowers the solid pressure.

Sensors measuring forces at the particle scale or the static pore pressure remains a challenge. However, both pressure-balanced and fully fluidized simulations show that local flow properties enable prediction of the basal pore pressure that matches experiments. Conversely, $P_{-} G$ (pore pressure inside the flow, which differs from the pressure measured at base of the flow) can be estimated from measurements of particle volumetric concentration and slip velocity. This could be a useful tool when dealing with small to large-scale experiments to get the pore pressure $\left(P_{-} G\right)$ of the basal portion of the flow, and also to estimate the (basal) fractional normal stress reduction (parameter " $N$ " in equation (40)).

The negative (relative to atmosphere) basal pore pressure signal implies that a particle located on top of a rough substrate bed would experience a lift force due to a vertical pore pressure gradient, which causes onset of uplift as shown in experiments (Roche et al., 2013). This process should occur for both pressure-balanced and fluidized flows and is highly dependent on the (basal) slip velocity. Once onset of uplift is promoted and the particle is incorporated at the flow base, additional upward lift forces due to internal shear and/or segregation effects can occur.

\subsection{Bidisperse Distributions and Implications for the Modeling of Natural Flows With Continuum Approaches}

Despite their small scale, present experiments and simulations span inertial numbers of 9 orders of magnitudes (Inertial numbers from $10^{-8}$ to 10; Figure 7), thus covering the fields of quasi-static regimes $(I<$ $\sim 0.001)$, intermediate $(\sim 0.001<I<\sim 0.1)$ and gaseous regimes $(I>\sim 0.1)$. The gaseous regime was the first to be accurately described (Bagnold, 1954, 1956) while the quasi-static and intermediate regimes have been increasingly better described in the past decade (GDR-MiDi, 2004; F. da Cruz et al., 2005; Zhang \& Kamrin, 2017). While in volcanology the Inertial number is not (generally) used to scale experiments, it is an essential parameter to ensure scaling of the mean particle rearrangement timescale over the flow deformation timescale and it was shown to control granular flow rheology (Andreotti et al., 2013; Forterre \& Pouliquen, 2008). While traditionally the inertial number is only used to describe spherical particles, recent studies suggest that it is valid also for nonspherical particles (Hidalgo et al., 2018; Nagy et al., 2017). Here, we assume 

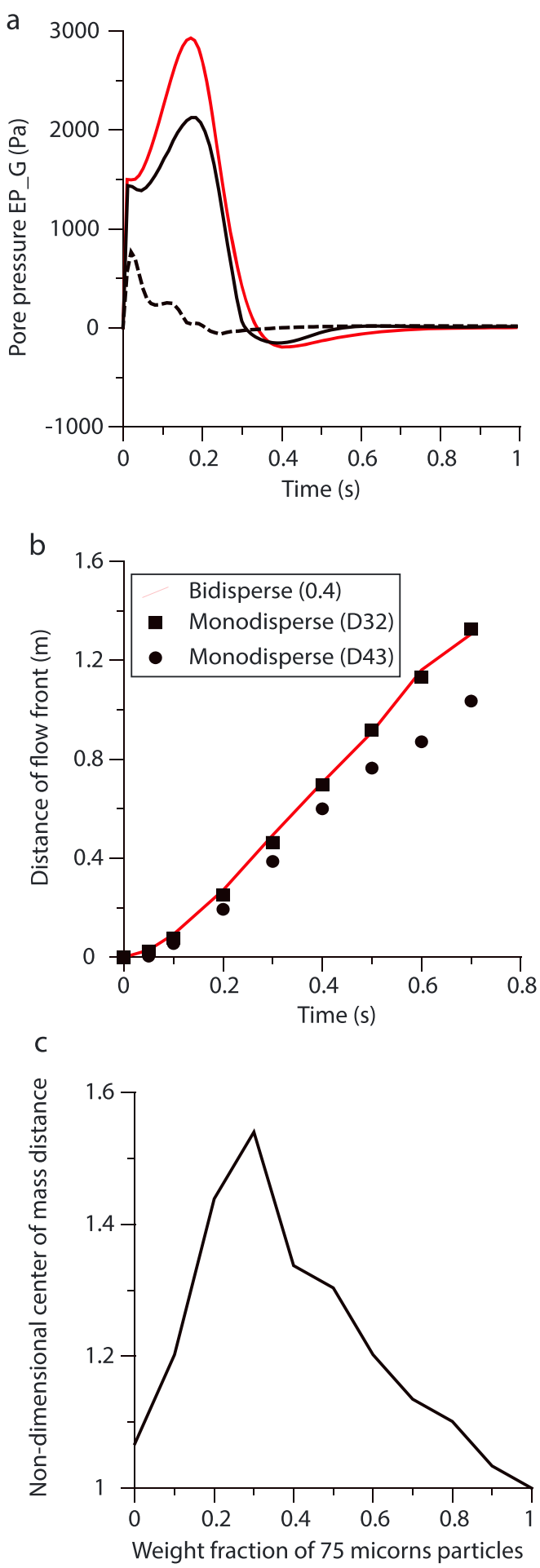

Figure 8. Pore pressure (at $0.0025 \mathrm{~m}$ of height) at $-0.125 \mathrm{~m}$ (in reservoir) for the bidisperse (red curve) mixture of $40 \mathrm{wt} . \% 75-\mu \mathrm{m}$ and $60 \mathrm{wt} . \% 700-\mu \mathrm{m}$ particles. Monodisperse simulations with similar initial parameters except for the mixture grain size of $160 \mu \mathrm{m}$ (D32, black line) and $500 \mu \mathrm{m}$ (D43, dashed line). (b) Flow front versus distance showing the overlap of the D32 simulation with the bidisperse simulation. (c) Distance of the center of mass of the flow at $1.2 \mathrm{~s}$ for various bidisperse mixtures of 75 and $800 \mu \mathrm{m}$. The distances were normalized to the distance of the mixture of $100 \% 75-\mu \mathrm{m}$ particles. that the inertial number can be used to describe unsteady concentrated volcanic mixtures, since the inertial number was successfully used in dam-break simulations to describe the transient granular rheology (Martin et al., 2017). We estimate the Inertial number (equation (3)) for an extreme case of a natural 10-m-thick highly sheared (shear rate $=$ $1,000 \mathrm{~s}^{-1}$ at the base) concentrated pyroclastic current of dense fragments with mean grain diameter of $0.01 \mathrm{~m}$ (solid density $=2,000 \mathrm{~kg} / \mathrm{m}^{3}$, solid concentration $=0.5$ ). The Inertial number is of the order of $10^{\circ}$, which is within the range investigated in this study. In fact, natural flows are expected to display Inertial numbers that tend to 0 (as shear goes to 0 ) and as high as $10^{2}$. Such high inertial numbers for dense pyroclastic density currents, however, require the solid pressure at the flow base to be low despite very high shear rates, which can be achieved if the flow weight is partially supported by pore pressure. Note that other scaling parameters would be needed in order to describe the unsteadiness or nonlocality of the granular rheology that could be at play in experimental and natural flows

In light of this scaling, we assume that most geological flows composed of gas and solid particles can be modeled following the same approach as described in benchtop simulations here. However, it is worth noting that benchtop experiments display a disproportionately little effect of pore fluid pressure compared to large-scale experiments or natural flows. For this reason, experiments and simulations were performed to fundamentally investigate the physics of granular flows when pore pressure is high, instead of predicting the runout of natural flows based on a drop height for instance.

One of the main challenges with multiphase simulations of natural scale, concentrated, granular flows is achieving sufficient resolution to resolve the mixture over large regions. This combination of high resolution and large domains requires significant computational resources. Here we used a grid size of the order of 5-10 particle diameters because the flow height at the front was of similar scale. The most important quantity is the number of cells per unit flow height. We showed in simulations that even with a flow described by as low as two to four cells (near flow front), the flow dynamics matched well experiments. Therefore, having a 10-m-thick flow described for instance by five cells (2-m resolution) mostly affects how the interface with the upper ash cloud is resolved. This will be investigated in future comparison between Pyroclastic flow eruption large-scale experiment (PELE) (New Zealand) and MFIX.

The particle size distribution of natural flows is never monodisperse. Here we illuminate the influence of bidispersity on the flow behavior by additional simulations of collapse of an expanded mixture (solid fraction = 0.57 ) of $40 \mathrm{wt} \%$ of $75-\mu \mathrm{m}$ glass beads with $60 \mathrm{wt} \%$ of $700-\mu \mathrm{m}$ glass beads, and by comparing with two simulations of monodisperse spheres (with same initial volume fraction) where the mean size used is the surface (Sauter) mean diameter D32 and the volume mean diameter D43. From the bidisperse distributions, the D32 and D43 were determined as

$$
D_{32}=\frac{1}{\sum \frac{x_{i}}{\bar{d}_{i}}} \text { and } D_{43}=\sum x_{i} d_{i}
$$

(Richardson et al., 2002), where $\bar{d}_{i}$ is the mean particle size is the sieve $i$, and $x_{i}$ is the fractional mass of particles of that sieve. 
Simulations with the D32 $(130 \mu \mathrm{m})$ mean diameter better predict the permeability of the mixture and lead to pore pressures similar to those of the bidisperse mixture while the column compacts (Figure 8a). As the flow defluidizes, the pore pressure diffuses at relatively similar rate in the D32 and bidisperse simulations. Note that the negative peak following the positive one is the result of the mixture dilation. Meanwhile, the D43 $(400 \mu \mathrm{m})$ mean diameter does not promote high pressure because of faster pressure diffusion and because of lower fluid drag (that impedes pore pressure formation) by orders of magnitude. This is not surprising as the permeability of the D43 (permeability $=2.4 \times 10^{-10} \mathrm{~m}^{2}$ ) mixture is $800 \%$ larger than the D32s (permeability $=2.9 \times 10^{-11} \mathrm{~m}^{2}$ ). The D32 characterizes the role of the mean aerodynamic drag of the particle size distribution whereas the D43 is a proxy for the prominent role of large particle in the contact network of the mixture. Using the latter mean diameter (to represent a polydisperse distribution) in continuum models such that using the $\mathrm{mu}(I)$ rheology has recently been shown to be the most accurate description of granular stresses (Gu et al., 2016). The very distinct permeability (8 times larger for D43 than for D42) and variable pore pressure has a strong influence on the flow kinematics, as shown in Figure 8b, where only the D32 simulation matches well the bidisperse counterpart.

Furthermore, we numerically simulated various blends of $75-$ and $700-\mu \mathrm{m}$ fractions from $0 \%$ to $100 \%$ and simulated the collapse of the pressure-balanced mixtures. The results illustrate the role of granular stresses with respect to grains size distribution. These simulations revealed that the 0.3 blend ( $30 \% 75 \mu \mathrm{m})$ leads to the optimum flow runout (Figure 8c). The overall behavior of the numerical flows replicates experimental observation of Roche et al. (2005) and Phillips et al. (2006), where increased mobility of the bidisperse mixture around $30 \%$ fine fraction is due to the formation of a lower region enriched in fines that "lubricate" the flow as they can roll on the substrate and by alleviating the energy dissipation through collisions (LinaresGuerrero et al., 2007). These observations are in line with the findings of Cagnoli and Piersanti (2015) who showed that fine-grained mixture dissipate energy per distance travel at a slower rate than coarser counterparts. This demonstrates the influence of size distribution on the dense flow dynamics. These results are encouraging and suggest the need to further focus experimental work on polydisperse flows (even with spheres) to better constrain the rheophysics of polydisperse mixtures. In particular, capturing the effect of grains angular shapes on energy dissipation rates is important as reported by DEM simulations (Cagnoli \& Piersanti, 2015; Mollon et al., 2015).

\section{Conclusion}

Continuum multiphase simulations of release of monodisperse, initially pressure-balanced piles of fine (75 $\mu \mathrm{m}$ ) particles suggest that a frictional model is necessary to capture granular stresses of high solid fraction flows, and the (Princeton) Srivastava-Sundaresan friction model is preferred over the Schaeffer model in the context of the MFIX code. With the Princeton frictional model, simulations match experiments well in terms of flow shape, flow kinematics and pore pressure. The latter forms even in initially pressurebalanced flows through dilation and compaction, which produce negative or positive pore pressure excursions, respectively. This suggests that pore pressure in pyroclastic flows with high amount of fine particles can arise through relative gas-particle motion. In particular, generation of positive pore pressure caused by rapid settling of material (e.g., Breard et al., 2017) is expected to occur at impact zones of collapsing pyroclastic fountains and/or in deflating pyroclastic density currents. Though internal or external sources of gas will further enhance pore pressure, these are not required to create fluidized pyroclastic mixtures. Simulations show that the negative basal pore pressure peak observed in many experiments forms when the dynamic pressure term exceeds the total pore pressure, which is primarily a function of the basal slip velocity. Using the local slip and solid fraction, we demonstrate that a basal pore pressure signal can be inverted to estimate the total pore pressure and fractional normal stress reduction in experiments. Even with the frictional model, flows remain in creeping motion at late stage, thus impeding "final" deposition. The latter is approximated by a criterion such that the inertial number $(<0.001)$ that characterizes regions of creep and near close packing.

Owing to the low mixture permeability and its high solid fraction, gas pore pressure can be retained for relatively long duration in initially fluidized flows, which propagate with a solid viscosity of nearly an order of magnitude lower than their initially pressure-balanced counterparts and hence have long runout distances. Finally, bidisperse simulations are also in agreement with similar experiments, and they show the role of 
particle size distribution on the flow permeability, which at the small scale dominates flow behavior over granular stresses and related particle size segregation.

Acknowledgments

Financial support was provided by the National Science Foundation grant EAR 1650382 (J. D.). We thank Roberto Sulpizio, Ben Andrews, and an anonymous reviewer for their thoughtful reviews. This is LabEx Clervolc contribution number 351. The data supporting the conclusions are shown in the figures and tables presented. On request the authors can also provide more specific results obtained from the experimental and numerical studies used to produce the figures.

\section{References}

Amarsid, L., Delenne, J. Y., Mutabaruka, P., Monerie, Y., Perales, F., \& Radjai, F. (2017). Viscoinertial regime of immersed granular flows. Physical Review E, 96(1), 012901. https://doi.org/10.1103/PhysRevE.96.012901

Andreotti, B., Forterre, Y., \& Pouliquen, O. (2013). Granular media: Between fluid and solid. Cambridge University Press, Cambridge. https://doi.org/10.1017/CBO9781139541008

Azéma, E., Estrada, N., \& Radjaï, F. (2012). Nonlinear effects of particle shape angularity in sheared granular media. Physical Review E, 86(4), 041301. https://doi.org/10.1103/PhysRevE.86.041301

Bagnold, R. A. (1954). Experiments on a gravity-free dispersion of large solid spheres in a Newtonian fluid under shear. Proceedings of the Royal Society of London - Series A, 225, 49-63.

Bagnold, R. A. (1956). The flow of cohesionless grains in fluid. Philosophical Transactions of the Royal Society of London, 249(964), 235-297. https://doi.org/10.1098/rsta.1956.0020

Balachandar, S., \& Eaton, J. K. (2010). Turbulent dispersed multiphase flow. Annual Review of Fluid Mechanics, 42(1), 111-133. https://doi. org/10.1146/annurev.fluid.010908.165243

Barker, T., Schaeffer, D. G., Bohorquez, P., \& Gray, J. M. N. T. (2015). Well-posed and ill-posed behaviour of the $\mu(I)$-rheology for granular flow. Journal of Fluid Mechanics, 779, 794-818. https://doi.org/10.1017/jfm.2015.412

Barker, T., Schaeffer, D. G., Shearer, M., \& Gray, J. M. N. T. (2017). Well-posed continuum equations for granular flow with compressibility and $\mu(I)$-rheology. Proceedings of the Royal Society A: Mathematical, Physical and Engineering Science, 473(2201). https://doi.org/10.1098/rspa.2016.0846

Bartelt, P., Valero, C. V., Feistl, T., Christen, M., Bühler, Y., \& Buser, O. (2017). Modelling cohesion in snow avalanche flow. Journal of Glaciology, 61(229), 837-850. https://doi.org/10.3189/2015JoG14J126

Benage, M. C., Dufek, J., \& Mothes, P. A. (2016). Quantifying entrainment in pyroclastic density currents from the Tungurahua eruption, Ecuador: Integrating field proxies with numerical simulations. Geophysical Research Letters, 43, 6932-6941. https://doi.org/10.1002/ 2016GL069527

Benyahia, S. (2008). Validation of two continuum granular flow theories. Industrial and Engineering Chemistry Research, 47(22), 8926-8932 . https://doi.org/10.1021/ie8003557

Benyahia, S., Syamlal, M., \& O'Brien, T. J. (2007). Study of the ability of multiphase continuum models to predict core-annulus flow. AIChE Journal, 53(10), 2549-2568. https://doi.org/10.1002/aic.11276

Bergantz, G. W., Schleicher, J. M., \& Burgisser, A. (2015). Open-system dynamics and mixing in magma mushes. Nature Geoscience, 8(10), 793-796. https://doi.org/10.1038/ngeo2534, https://www.nature.com/articles/ngeo2534\#supplementary-information

Beverloo, W. A., Leniger, H. A., \& van de Velde, J. (1961). The flow of granular solids through orifices. Chemical Engineering Science, 15(3-4), 260-269. https://doi.org/10.1016/0009-2509(61)85030-6

Boyer, F., Guazzelli, É., \& Pouliquen, O. (2011). Unifying suspension and granular rheology. Physical Review Letters, 107(18), 188301. https://doi.org/10.1103/PhysRevLett.107.188301

Breard, E., Lube, G., Jones, J. R., Dufek, J., Cronin, S. J., Valentine, G. A., \& Moebis, A. (2016). Coupling of turbulent and non-turbulent flow regimes within pyroclastic density currents. Nature Geoscience, 9(10), 767-771. https://doi.org/10.1038/ngeo2794, http://www. nature.com/ngeo/journal/v9/n10/abs/ngeo2794.html\#supplementary-information

Breard, E. C. P., Dufek, J., \& Lube, G. (2017). Enhanced mobility in concentrated pyroclastic density currents: An examination of a selffluidization mechanism. Geophysical Research Letters, 45, 654-664. https://doi.org/10.1002/2017GL075759

Breard, E. C. P., \& Lube, G. (2017). Inside pyroclastic density currents-Uncovering the enigmatic flow structure and transport behaviour in large-scale experiments. Earth and Planetary Science Letters, 458, 22-36. https://doi.org/10.1016/j.epsl.2016.10.016

Britter, R. E., \& Linden, P. F. (1980). The motion of the front of a gravity current travelling down an incline. Journal of Fluid Mechanics, 99(3), 531-543. https://doi.org/10.1017/S0022112080000754

Brodu, N., Delannay, R., Valance, A., \& Richard, P. (2015). New patterns in high-speed granular flows. Journal of Fluid Mechanics, 769, 218-228. https://doi.org/10.1017/jfm.2015.109

Cagnoli, B., \& Piersanti, A. (2015). Grain size and flow volume effects on granular flow mobility in numerical simulations: 3-D discrete element modeling of flows of angular rock fragments. Journal of Geophysical Research: Solid Earth, 120, 2350-2366. https://doi.org/ 10.1002/2014JB011729

Carnahan, N. F., \& Starling, K. E. (1969). Equation of state for nonattracting rigid spheres. The Journal of Chemical Physics, 51(2), 635-636. https://doi.org/10.1063/1.1672048

Cassar, C., Nicolas, M., \& Pouliquen, O. (2005). Submarine granular flows down inclined planes. Physics of Fluids, 17(10), 103301. https:// doi.org/10.1063/1.2069864

Charbonnier, S. J., \& Gertisser, R. (2011). Deposit architecture and dynamics of the 2006 block-and-ash flows of Merapi Volcano, Java, Indonesia. Sedimentology, 58(6), 1573-1612. https://doi.org/10.1111/j.1365-3091.2011.01226.x

Chialvo, S., \& Sundaresan, S. (2013). A modified kinetic theory for frictional granular flows in dense and dilute regimes. Physics of Fluids, 25(7), 070603. https://doi.org/10.1063/1.4812804

Courrech du Pont, S., Gondret, P., Perrin, B., \& Rabaud, M. (2003). Granular avalanches in fluids. Physical Review Letters, $90(4), 044301$. https://doi.org/10.1103/PhysRevLett.90.044301

da Cruz, F., Emam, S., Prochnow, M., Roux, J.-N., \& Chevoir, F. (2005). Rheophysics of dense granular materials: Discrete simulation of plane shear flows. Physical Review E, 72(2), 021309. https://doi.org/10.1103/PhysRevE.72.021309

Dartevelle, S. (2007), From model conception to verification and validation, a global approach to multiphase Navier-Stoke models with an emphasis on volcanic explosive phenomenology, United States. Department of Energy.

Dartevelle, S., \& Valentine, G. A. (2008). Multiphase magmatic flows at Yucca Mountain, Nevada. Journal of Geophysical Research, 113, B12209. https://doi.org/10.1029/2007JB005367

Drucker, D. C., \& Prager, W. (1952). Soil mechanics and plastic analysis for limit design. Quarterly of Applied Mathematics, 10(2), 157-165. https://doi.org/10.1090/qam/48291

Druitt, T. H. (1998). Pyroclastic density currents. Geological Society Special Publication, 145(1), 145-182. https://doi.org/10.1144/GSL. SP.1996.145.01.08 
Du, W., Wang, D., \& Yang, Y. (2016). Effect of variable particle stiffness on force propagation and mechanical response of a composite granular material. European Physical Journal E: Soft Matter and Biological Physics, 39(6), 60. https://doi.org/10.1140/epje/i201616060-3

Duan, Y., Feng, Z.-G., Michaelides, E. E., \& Mao, S. (2017). Modified kinetic theory applied to the shear flows of granular materials. Physics of Fluids, 29(4), 043302. https://doi.org/10.1063/1.4979632

Dufek, J. (2016). The fluid mechanics of pyroclastic density currents. Annual Review of Fluid Mechanics, 48(1), 459-485. https://doi.org/ 10.1146/annurev-fluid-122414-034252

Dufek, J., \& Bergantz, G. W. (2005). Transient two-dimensional dynamics in the upper conduit of a rhyolitic eruption; a comparison of closure models for the granular stress. Journal of Volcanology and Geothermal Research, 143(1-3), 113-132. https://doi.org/10.1016/j. jvolgeores.2004.09.013

Dufek, J., \& Bergantz, G. W. (2007). Dynamics and deposits generated by the Kos Plateau Tuff eruption; controls of basal particle loss on pyroclastic flow transport. Geochemistry, Geophysics, Geosystems, 8, Q12007. https://doi.org/10.1029/2007GC001741

Dufek, J., Wexler, J., \& Manga, M. (2009). Transport capacity of pyroclastic density currents: Experiments and models of substrate-flow interaction. Journal of Geophysical Research, 114, B11203. https://doi.org/10.1029/2008JB006216

Dunatunga, S., \& Kamrin, K. (2015). Continuum modelling and simulation of granular flows through their many phases. Journal of Fluid Mechanics, 779, 483-513. https://doi.org/10.1017/jfm.2015.383

Esposti Ongaro, T., Clarke, A. B., Voight, B., Neri, A., \& Widiwijayanti, C. (2012). Multiphase flow dynamics of pyroclastic density currents during the May 18, 1980 lateral blast of Mount St. Helens. Journal of Geophysical Research, 117, B06208. https://doi.org/10.1029/ 2011JB009081

Folk, R. L., \& Ward, W. C. (1957). Brazos River bar [Texas]; a study in the significance of grain size parameters. Journal of Sedimentary Petrology, 27(1), 3-26. https://doi.org/10.1306/74D70646-2B21-11D7-8648000102C1865D

Forterre, Y., \& Pouliquen, O. (2008). Flows of dense granular media. Annual Review of Fluid Mechanics, 40(1), 1-24. https://doi.org/ 10.1146/annurev.fluid.40.111406.102142

Fullard, L., Breard, E., Davies, C., Lagrée, P.-Y., Popinet, S., \& Lube, G. (2017). Testing the $\mu(I)$ granular rheology against experimental silo data. EPJ Web of Conferences, 140, 11002. https://doi.org/10.1051/epjconf/201714011002

GDR-MiDi (2004). On dense granular flows. European Physical Journal E: Soft Matter and Biological Physics, 14(4), 341-365. https://doi. org/10.1140/epje/i2003-10153-0

Gera, D., Syamlal, M., \& O'Brien, T. (2004). Hydrodynamics of particle segregation in fluidized beds. International Journal of Multiphase Flow, 30(4), 419-428. https://doi.org/10.1016/j.ijmultiphaseflow.2004.01.003

Goddard, J. D. (2014). PDF Continuum Modeling of Granular Media. Applied Mechanics Reviews, 66(5), 050801. https://doi.org/10.1115/ 1.4026242

Goldhirsch, I. (2003). Rapid granular flows. Annual Review of Fluid Mechanics, 35(1), 267-293. https://doi.org/10.1146/annurev. fluid.35.101101.161114

Gu, Y., Ozel, A., \& Sundaresan, S. (2016). Rheology of granular materials with size distributions across dense-flow regimes. Powder Technology, 295(Supplement C, 322-329. https://doi.org/10.1016/j.powtec.2016.03.035

Gueugneau, V., Kelfoun, K., Roche, O., \& Chupin, L. (2017). Effects of pore pressure in pyroclastic flows: Numerical simulation and experimental validation. Geophysical Research Letters, 44, 2194-2202. https://doi.org/10.1002/2017GL072591

Hidalgo, R. C., Szabó, B., Gillemot, K., Börzsönyi, T., \& Weinhart, T. (2018). Rheological response of nonspherical granular flows down an incline. Physical Review Fluids, 3(7), 074301. https://doi.org/10.1103/PhysRevFluids.3.074301

Holyoake, A. J., \& McElwaine, J. N. (2012). High-speed granular chute flows. Journal of Fluid Mechanics, 710, 35-71. https://doi.org/ $10.1017 / \mathrm{jfm} .2012 .331$

Ionescu, I. R., Mangeney, A., Bouchut, F., \& Roche, O. (2015). Viscoplastic modeling of granular column collapse with pressure-dependent rheology. Journal of Non-Newtonian Fluid Mechanics, 219, 1-18. https://doi.org/10.1016/j.jnnfm.2015.02.006

Iverson, R. M. (2005). Regulation of landslide motion by dilatancy and pore pressure feedback. Journal of Geophysical Research, 110(F2). https://doi.org/10.1029/2004jf000268

Iverson, R. H. (1997). The physics of debris flows. Reviews of Geophysics, 35(3), 245-296. https://doi.org/10.1029/97RG00426

Jaeger, H. M., Nagel, S. R., \& Behringer, R. P. (1996). Granular solids, liquids, and gases. Reviews of Modern Physics, 68(4), $1259-1273$. https://doi.org/10.1103/RevModPhys.68.1259

Jenike, A. W. (1987). A theory of flow of particulate solids in converging and diverging channels based on a conical yield function. Powder Technology, 50(3), 229-236. https://doi.org/10.1016/0032-5910(87)80068-2

Jenkins, J. T., \& Berzi, D. (2010). Dense inclined flows of inelastic spheres: Tests of an extension of kinetic theory. Granular Matter, 12(2), 151-158. https://doi.org/10.1007/s10035-010-0169-8

Jenkins, J. T., \& Savage, S. B. (1983). A theory for the rapid flow of identical, smooth, nearly elastic, spherical particles. Journal of Fluid Mechanics, 130(1), 187-202. https://doi.org/10.1017/S0022112083001044

Jenkins, J. T., \& Zhang, C. (2002). Kinetic theory for identical, frictional, nearly elastic spheres. Physics of Fluids, 14(3), 1228-1235. https:// doi.org/10.1063/1.1449466

Johson, P. C., \& Jackson, R. (1987). Frictional-collisional constitutive relations for the granular materials, with application to plane shearing. Journal of Fluid Mechanics, (145), 223-233.

Jop, P., Forterre, Y., \& Pouliquen, O. (2006). A constitutive law for dense granular flows. Nature, 441(7094), 727-730. https://doi.org/ 10.1038 /nature04801

Kamrin, K. (2017). A hierarchy of granular continuum models: Why flowing grains are both simple and complex. EPJ Web of Conferences, 140, 01007. https://doi.org/10.1051/epjconf/201714001007

Kamrin, K., \& Koval, G. (2012). Nonlocal constitutive relation for steady granular flow. Physical Review Letters, 108(17), 178301. https://doi. org/10.1103/PhysRevLett.108.178301

Lagrée, P. Y., Staron, L., \& Popinet, S. (2011). The granular column collapse as a continuum: Validity of a two-dimensional Navier-Stokes model with a $\mu(I)$-rheology. Journal of Fluid Mechanics, 686, 378-408. https://doi.org/10.1017/jfm.2011.335

Li, T. W., Rogers, W. A., Syamlal, M., Dietiker, J. F., Musser, J., Shahnam, M., \& Rabha, S. (2017). The NETL MFiX Suite of multiphase flow models: A brief review and recent applications of MFiX-TFM to fossil energy technologies. Chemical Engineering Science, 169, 259-272. https://doi.org/10.1016/j.ces.2016.07.043

Linares-Guerrero, E., Goujon, C., \& Zenit, R. (2007). Increased mobility of bidisperse granular avalanches. Journal of Fluid Mechanics, 593, 475-504. https://doi.org/10.1017/S0022112007008932 
Lube, G., Breard, E. C. P., Jones, J. R., Fullard, L., Dufek, J., Cronin Shane, J., et al. (2019). The lubrication of pyroclastic flows. Nature of Geoscience.

Lun, C. K. K., Savage, S. B., Jeffrey, D. J., \& Chepurniy, N. (1984). Kinetic theories for granular flow: Inelastic particles in Couette flow and slightly inelastic particles in a general flowfield. Journal of Fluid Mechanics, 140(1), 223-256. https://doi.org/10.1017/ S0022112084000586

Lusso, C., Ern, A., Bouchut, F., Mangeney, A., Farin, M., \& Roche, O. (2017). Two-dimensional simulation by regularization of free surface viscoplastic flows with Drucker-Prager yield stress and application to granular collapse. Journal of Computational Physics, 333, 387-408. https://doi.org/10.1016/j.jcp.2016.12.036

Marino, B. M., Thomas, L. P., \& Linden, P. F. (2005). The front condition for gravity currents. Journal of Fluid Mechanics, 536, 49-78. https://doi.org/10.1017/S0022112005004933

Martin, N., Ionescu, I. R., Mangeney, A., Bouchut, F., \& Farin, M. (2017). Continuum viscoplastic simulation of a granular column collapse on large slopes: $\mu(I)$ rheology and lateral wall effects. Physics of Fluids, 29(1), 013301. https://doi.org/10.1063/1.4971320

Maurin, R., Chauchat, J., Chareyre, B., \& Frey, P. (2015). A minimal coupled fluid-discrete element model for bedload transport. Physics of Fluids, 27(11), 113302. https://doi.org/10.1063/1.4935703

Meiburg, E., \& Kneller, B. (2010). Turbidity currents and their deposits. Annual Review of Fluid Mechanics, 42(1), 135-156. https://doi.org/ 10.1146/annurev-fluid-121108-145618

Mollon, G., Richefeu, V., Villard, P., \& Daudon, D. (2015). Discrete modelling of rock avalanches: Sensitivity to block and slope geometries. Granular Matter, 17(5), 645-666. https://doi.org/10.1007/s10035-015-0586-9

Nagy, D. B., Claudin, P., Börzsönyi, T., \& Somfai, E. (2017). Rheology of dense granular flows for elongated particles. Physical Review E, 96(6), 062903. https://doi.org/10.1103/PhysRevE.96.062903

Phillips, J. C., Hogg, A. J., Kerswell, R. R., \& Thomas, N. H. (2006). Enhanced mobility of granular mixtures of fine and coarse particles. Earth and Planetary Science Letters, 246(3-4), 466-480. https://doi.org/10.1016/j.epsl.2006.04.007

Pouliquen, O. (1999). Scaling laws in granular flows down rough inclined planes. Physics of Fluids, 11(3), 542-548. https://doi.org/10.1063/ 1.869928

Pouliquen, O., \& Chevoir, F. (2002). Dense flows of dry granular material. Comptes Rendus Physique, 3(2), 163-175. https://doi.org/10.1016/ S1631-0705(02)01309-9

Richardson, J. F., Harker, J. H., \& Backhurst, J. R. (Eds.) (2002). Chapter 1-Particulate solids, in Chemical engineering (5th ed., pp. 1-94) Butterworth-Heinemann, Oxford. https://doi.org/10.1016/B978-0-08-049064-9.50012-6

Roche, O. (2012). Depositional processes and gas pore pressure in pyroclastic flows: An experimental perspective. Bulletin of Volcanology, 74(8), 1807-1820. https://doi.org/10.1007/s00445-012-0639-4

Roche, O., Buesch, D. C., \& Valentine, G. A. (2016). Slow-moving and far-travelled dense pyroclastic flows during the Peach Spring supereruption. Nature Communications, 7(1). https://doi.org/10.1038/ncomms10890

Roche, O., Gilbertson, M. A., Phillips, J. C., \& Sparks, R. S. J. (2005). Inviscid behaviour of fines-rich pyroclastic flows inferred from experiments on gas-particle mixtures. Earth and Planetary Science Letters, 240(2), 401-414. https://doi.org/10.1016/j. epsl.2005.09.053

Roche, O., Gilbertson, M. A., Phillips, J. C., \& Sparks, R. S. J. (2006). The influence of particle size on the flow of initially fluidised powders Powder Technology, 166(3), 167-174. https://doi.org/10.1016/j.powtec.2006.05.010

Roche, O., Montserrat, S., Niño, Y., \& Tamburrino, A. (2008). Experimental observations of water-like behavior of initially fluidized, dam break granular flows and their relevance for the propagation of ash-rich pyroclastic flows. Journal of Geophysical Research, 113, B12203. https://doi.org/10.1029/2008JB005664

Roche, O., Montserrat, S., Niño, Y., \& Tamburrino, A. (2010). Pore fluid pressure and internal kinematics of gravitational laboratory airparticle flows: Insights into the emplacement dynamics of pyroclastic flows. Journal of Geophysical Research, 115, B09206. https://doi. org/10.1029/2009JB007133

Roche, O., Nino, Y., Mangeney, A., Brand, B., Pollock, N., \& Valentine, G. A. (2013). Dynamic pore-pressure variations induce substrate erosion by pyroclastic flows. Geology, 41(10), 1107-1110. https://doi.org/10.1130/G34668.1

Rognon, P. G., Roux, J. N., Wolf, D., Naaïm, M., \& Chevoir, F. (2006). Rheophysics of cohesive granular materials. EPL (Europhysics Letters), 74(4), 644-650. https://doi.org/10.1209/epl/i2005-10578-y

Roux, J.-N., \& Combe, G. (2002). Quasistatic rheology and the origins of strain. Comptes Rendus Physique, 3(2), 131-140. https://doi.org/ 10.1016/S1631-0705(02)01306-3

Roux, R., \& Radjai, F. (1998). Physics of dry granular media, (pp. 229-236). Springer.

Rowley, P., Roche, O., Druitt, T., \& Cas, R. (2014). Experimental study of dense pyroclastic density currents using sustained, gas-fluidized granular flows. Bulletin of Volcanology, 76(9), 1-13. https://doi.org/10.1007/s00445-014-0855-1

Savage, S. B. (1998). Analyses of slow high-concentration flows of granular materials. Journal of Fluid Mechanics, 377, 1-26. https://doi.org/ $10.1017 /$ S0022112098002936

Schaeffer, D. G. (1987). Instability in the evolution equations describing incompressible granular flow. Journal of Differential Equations, 66(1), 19-50. https://doi.org/10.1016/0022-0396(87)90038-6

Shin, J. O., Dalziel, S. B., \& Linden, P. F. (2004). Gravity currents produced by lock exchange. Journal of Fluid Mechanics, 521, 1-34. https:// doi.org/10.1017/S002211200400165X

Sparks, R. S. J. (1976). Grain size variations in ignimbrites and implications for the transport of pyroclastic flows. Sedimentology, 23(2), 147-188. https://doi.org/10.1111/j.1365-3091.1976.tb00045.x

Sparks, R. S. J. (1978). Gas release rates from pyroclastic flows: A assessment of the role of fluidisation in their emplacement. Bulletin Volcanologique, 41(1), 1-9. https://doi.org/10.1007/BF02597679

Srivastava, A., \& Sundaresan, S. (2003). Analysis of a frictional-kinetic model for gas-particle flow. Powder Technology, 129(1-3), 72-85. https://doi.org/10.1016/S0032-5910(02)00132-8

Staron, L., Lagrée, P. Y., \& Popinet, S. (2014). Continuum simulation of the discharge of the granular silo. European Physical Journal E: Soft Matter and Biological Physics, 37(1), 5. https://doi.org/10.1140/epje/i2014-14005-6.

Stefan, L. (2009). Towards dense, realistic granular media in 2D. Nonlinearity, 22(12), R101.

Sulpizio, R., P, D., D. M, D., \& D, S. (2014). Pyroclastic density currents; state of the art and perspectives. Journal of Volcanology and Geothermal Research, 283, 36-65.

Sweeney, M. R., \& Valentine, G. A. (2017). Impact zone dynamics of dilute mono- and polydisperse jets and their implications for the initial conditions of pyroclastic density currents. Physics of Fluids, 29(9), 093304. https://doi.org/10.1063/1.5004197

Syamlal, M. (1985). Multiphase hydrodynamics of gas-solid flow, Illinois Insititute of Technology, Chicago. 
Syamlal, M., Celik, I. B., \& Benyahia, S. (2017). Quantifying the uncertainty introduced by discretization and time-averaging in two-fluid model predictions. AIChE Journal, 63(12), 5343-5360. https://doi.org/10.1002/aic.15868

Syamlal, M., Musser, J., \& Dietiker, J.-F. (2016). The two-fluid model in MFIX. In E. E. Michaelides, C. T. Crowe, \& J. D. Schwarzkopf (Eds.), Multiphase flow handbook, (2nd ed.pp. 242-274). Boca Raton, Florida, USA: CRC Press.

Syamlal, M. \& Pannala, S. (2011). Multiphase continuum formulation for gas-solid reacting flows. In M. S. a. T. J. O. B. E. S. Pannala (Ed.), Cumputational gas-solids flows and reacting systems: Theory, methods and practice (pp. 1-65). Hershey, PA: IGI Gloabla.

Syamlal, M. W. R., \& O'Brien, T. J. (1993). MFIX documentation: Theory guide, (p. 49 pp., DOE/METC-94/1004, DE9400,097). Washington, D. C: U.S. Dep. of Energy.

Tardos, G. I. (1997). A fluid mechanistic approach to slow, frictional flow of powders. Powder Technology, 92(1), 61-74. https://doi.org/ 10.1016/S0032-5910(97)03218-X

Valentine, G. A., \& Sweeney, M. R. (2018). Compressible flow phenomena at inception of lateral density currents fed by collapsing gasparticle mixtures. Journal of Geophysical Research: Solid Earth, 123, 1286-1302. https://doi.org/10.1002/2017JB015129

Vescovi, D., Berzi, D., Richard, P., \& Brodu, N. (2014). Plane shear flows of frictionless spheres: Kinetic theory and 3D soft-sphere discrete element method simulations. Physics of Fluids, 26(5), 053305. https://doi.org/10.1063/1.4879267

Wood, D. M. (1990). Soil behaviour and critical state soil mechanics. Cambridge: Cambridge university press.

Zhang, Q., \& Kamrin, K. (2017). Microscopic description of the granular fluidity field in nonlocal flow modeling. Physical Review Letters, 118(5), 058001. https://doi.org/10.1103/PhysRevLett.118.058001

Zhou, Y. C., Xu, B. H., Yu, A. B., \& Zulli, P. (2001). Numerical investigation of the angle of repose of monosized spheres. Physical Review E, 64(2), 021301. https://doi.org/10.1103/PhysRevE.64.021301 